\title{
On the Existence of Equilibrium Points, Boundedness, Oscillating Behavior and Positivity of a SVEIRS Epidemic Model under Constant and Impulsive Vaccination
}

\author{
M. De la Sen, ${ }^{1,2}$ Ravi P. Agarwal, ${ }^{3,4}$ A. Ibeas, ${ }^{5}$ \\ and S. Alonso-Quesada ${ }^{1,2}$ \\ ${ }^{1}$ Institute of Research and Development of Processes, Faculty of Science and Technology, \\ University of the Basque Country, P.O. Box 644, 48080 Bilbao, Spain \\ ${ }^{2}$ Department of Electricity and Electronics, Faculty of Science and Technology, \\ University of the Basque Country, P.O. Box 644, 48080 Bilbao, Spain \\ ${ }^{3}$ Department of Mathematical Sciences, Florida Institute of Technology, \\ Melbourne, FL 32901, USA \\ ${ }^{4}$ Mathematics and Statistics Department, King Fahd University of Petroleum and Minerals, \\ Dhahran 31261, Saudi Arabia \\ ${ }^{5}$ Department of Telecommunications and Systems Engineering, Universitat Autònoma de Barcelona, \\ 08193 Bellaterra, Barcelona, Spain
}

Correspondence should be addressed to M. De la Sen, manuel.delasen@ehu.es

Received 17 January 2011; Accepted 23 February 2011

Academic Editor: Claudio Cuevas

Copyright (C) 2011 M. De la Sen et al. This is an open access article distributed under the Creative Commons Attribution License, which permits unrestricted use, distribution, and reproduction in any medium, provided the original work is properly cited.

This paper discusses the disease-free and endemic equilibrium points of a SVEIRS propagation disease model which potentially involves a regular constant vaccination. The positivity of such a model is also discussed as well as the boundedness of the total and partial populations. The model takes also into consideration the natural population growing and the mortality associated to the disease as well as the lost of immunity of newborns. It is assumed that there are two finite delays affecting the susceptible, recovered, exposed, and infected population dynamics. Some extensions are given for the case when impulsive nonconstant vaccination is incorporated at, in general, an aperiodic sequence of time instants. Such an impulsive vaccination consists of a culling or a partial removal action on the susceptible population which is transferred to the vaccinated one. The oscillatory behavior under impulsive vaccination, performed in general, at nonperiodic time intervals, is also discussed.

\section{Introduction}

Important control problems nowadays related to Life Sciences are the control of ecological models like, for instance, those of population evolution (Beverton-Holt model, Hassell model, 
Ricker model, etc. [1-5]) via the online adjustment of the species environment carrying capacity, that of the population growth or that of the regulated harvesting quota as well as the disease propagation via vaccination control. In a set of papers, several variants and generalizations of the Beverton-Holt model (standard time-invariant, time-varying parameterized, generalized model or modified generalized model) have been investigated at the levels of stability, cycle-oscillatory behavior, permanence, and control through the manipulation of the carrying capacity (see, e.g., [1-5]). The design of related control actions has been proved to be important in those papers at the levels, for instance, of aquaculture exploitation or plague fighting. On the other hand, the literature about epidemic mathematical models is exhaustive in many books and papers. A nonexhaustive list of references is given in this manuscript, compare [6-14] (see also the references listed therein). The sets of models include the most basic ones, [6, 7].

(i) SI-models where not removed-by-immunity population is assumed. In other words, only susceptible and infected populations are assumed.

(ii) SIR-models, which include susceptible, infected, and removed-by-immunity populations.

(iii) SEIR-models where the infected populations is split into two ones (i.e., the "infected" which incubate the disease but do not still have any disease symptoms and the "infectious" or "infective" which do exhibit the external disease symptoms).

The three above models have two possible major variants, namely, the so-called "pseudomass action models," where the total population is not taken into account as a relevant disease contagious factor or disease transmission power, and the so-called "true-mass action models", where the total population is more realistically considered as being an inverse factor of the disease transmission rates. There are other many variants of the above models, for instance, including vaccination of different kinds: constant [8], impulsive [12], discretetime, and so forth, incorporating point or distributed delays [12, 13], oscillatory behaviors [14], and so forth. On the other hand, variants of such models become considerably simpler for the disease transmission among plants [6, 7]. Some generalizations involve the use of a mixed regular continuous-time/impulsive vaccination control strategies for generalized time-varying epidemic model which is subject to point and distributed time-varying delays, $[12,13,15-17]$. Other well-known types of epidemic models are the so-called SVEIRS epidemic models which incorporate the dynamics of a vaccinated population, and the "infected" population without external symptoms of the SEIR-type models is replaced with an "exposed" population subject to a certain dynamics, $[18,19]$. Thus, in the context of SVEIRS models, the infected and infectious populations of the SEIR models are joined in a single "infected" population $I(t)$ while there is an exposed population $E(t)$ present in the model. In this paper, we focus on the existence and some properties of disease-free and endemic equilibrium points of a SVEIRS model subject to an eventual constant regular vaccination rather than to an impulsive vaccination type. Some issues about boundedness and positivity of the model are also investigated. The following impulsive-free SVEIRS epidemic model, of a modified true-mass action type, with regular constant vaccination is being firstly considered

$$
\begin{gathered}
\dot{S}(t)=b(1-S(t))-\beta \frac{S(t) I(t)}{1+\eta S(t)}+\gamma I(t-\omega) e^{-b \omega}+v\left(1-V_{c}\right) N(t), \\
\dot{V}(t)=-\frac{\delta \beta V(t) I(t)}{1+\eta V(t)}-\left(\gamma_{1}+b\right) V(t)+v V_{c} N(t),
\end{gathered}
$$




$$
\begin{gathered}
E(t)=\beta \int_{t-\omega}^{t}\left(\frac{S(u) I(u)}{1+\eta S(u)}+\frac{\delta V(u) I(u)}{1+\eta V(u)}\right) e^{-b(t-u)} d u, \\
\dot{I}(t)=\beta e^{-b \tau}\left(\frac{S(t-\tau)}{1+\eta S(t-\tau)}+\frac{\delta V(t-\tau)}{1+\eta V(t-\tau)}\right) I(t-\tau)-(\gamma+b+\alpha) I(t), \\
\dot{R}(t)=-b R(t)+\gamma_{1} V(t)+\gamma\left(I(t)-I(t-\omega) e^{-b \omega}\right),
\end{gathered}
$$

where $S, V, E, I$, and $R$ are, respectively, the susceptible, vaccinated, exposed, infected (or infective or infectious), and recovered populations, $N(t)$ is the total population being the sum of the above ones, and $V_{c} \in[0,1]$ is a constant vaccination action. There are potential latent and immune periods denoted by $\tau$ and $\omega$, respectively, which are internal delays in the dynamic system (1.1)-(1.5), and $b$ is the natural birth rate and death rate of the population. The parameter $v<b$ takes into account a vaccination action on newborns which decreases the incremental susceptible population through time, $\gamma_{1}$ is the average rate for vaccines to obtain immunity and move into recovered population, and $\beta$ (disease transmission constant) and $\delta \beta$ are, respectively, average numbers for contacts of an infective with a susceptible and an infective with a vaccinated individual per unit of time, [18]. The periodic impulsive, rather than regular, vaccination action proposed in $[18,19]$, can be got from (1.1)-(1.5) with $V_{c}=0$ while adding either corresponding "culling" action, or, alternatively, a less drastic "partial removal of susceptible from the habitat" action. This implies in practical terms to put in quarantine a part of the susceptible population so as to minimize the effects of the disease propagation what corresponds with a population decrease of the susceptible in the habitat under study and a parallel increase of the vaccinated populations at times being an integer multiple of some prefixed period $T>0$. This paper investigate through Sections 2 4 the existence and uniqueness of the delay-free and endemic equilibrium points as well as the positivity and boundedness of the state-trajectory solutions under arbitrary nonnegative initial conditions and optional constant vaccination. Some generalized extensions concerning this model are given in Section 5 by using aperiodic impulsive vaccination with time-varying associated gains, in general, and investigating the state-trajectory solution properties. This impulsive vaccinations strategy will be performed as follows at a sequence of time instants $\left\{t_{k}\right\}_{k \in \mathbf{Z}_{0+}}$ ran in general at a nonperiodic "in-between" sampling interval sequence:

$$
\begin{aligned}
& S\left(t_{k}^{+}\right)=\left(1-\theta_{k}\right) S\left(t_{k}\right) ; \quad V\left(t_{k}^{+}\right)=V\left(t_{k}\right)+\theta_{k} S\left(t_{k}\right), \\
& E\left(t_{k}^{+}\right)=E\left(t_{k}\right) ; \quad I\left(t_{k}^{+}\right)=I\left(t_{k}\right) ; \quad R\left(t_{k}^{+}\right)=R\left(t_{k}\right) .
\end{aligned}
$$

Examples are provided in Section 6. It has to be pointed out that other variants of epidemic models have been recently investigated as follows. In [20], a mixed regular and impulsive vaccination action is proposed for a SEIR epidemic mode model which involves also mixed point and distributed delays. In [21], an impulsive vaccination strategy is discussed for a SVEIR epidemic model whose latent period is a point delay while the existence of an immune period is not assumed. In [22], a latent period is introduced in the susceptible population of a SIR epidemic model with saturated incidence rate. The disease-free equilibrium point results to be locally asymptotically stable if the reproduction number is less than unity while the endemic equilibrium point is locally asymptotically stable if such a number exceeds unity. 


\section{The Disease-Free Equilibrium Point}

The potential existence of a disease-free equilibrium point is now discussed which asymptotically removes the disease if $v<b$.

Theorem 2.1. Assume that $v<b$. Then, the disease-free equilibrium point $E^{*}=I^{*}=0$ fulfils

$$
\begin{gathered}
R^{*}=\frac{v \gamma_{1} V_{c} N^{*}}{b\left(\gamma_{1}+b\right)}=\gamma_{1} \frac{\left(b-v\left(1-V_{c}\right)\right) N^{*}-b}{\left(\gamma_{1}+b\right) b}, \\
V^{*}=\frac{v V_{c} N^{*}}{\gamma_{1}+b}=\frac{\left(b-v\left(1-V_{c}\right)\right) N^{*}-b}{\gamma_{1}+b}, \quad S^{*}=1+\frac{v N^{*}\left(1-V_{c}\right)}{b},
\end{gathered}
$$

which imply the following further constraints:

$$
N^{*}=\frac{b}{b-v^{\prime}}, \quad S^{*}-1=\frac{v\left(1-V_{c}\right)}{b-v}, \quad V^{*}+R^{*}=\frac{v V_{c} N^{*}}{b}=\frac{v V_{c}}{b-v} .
$$

Two particular disease-free equilibrium points are $S^{*}=N^{*}=b /(b-v), E^{*}=I^{*}=V^{*}=R^{*}=0$ if $V_{c}=0$, and $S^{*}=1, V^{*}=v N^{*} /\left(\gamma_{1}+b\right)=v b /\left(\gamma_{1}+b\right)(b-v), R^{*}=v \gamma_{1} /\left(\gamma_{1}+b\right)(b-v), E^{*}=I^{*}=0$ if $V_{c}=1$.

$$
\text { If } v \geq b \text {, then there is no disease-free equilibrium points. }
$$

Proof. Any existing equilibrium points are calculated as follows by zeroing (1.1), (1.2), (1.4), and (1.5) and making (1.3) identical to an equilibrium value $E^{*}$ what leads to:

$$
\begin{gathered}
b-\left(b+\frac{\beta I^{*}}{1+\eta S^{*}}\right) S^{*}+\gamma I^{*} e^{-b \omega}+v N^{*}\left(1-V_{c}\right)=0, \\
-\left(\frac{\delta \beta I^{*}}{1+\eta V^{*}}+\gamma_{1}+b\right) V^{*}+v N^{*} V_{c}=0, \\
E^{*}=\frac{\beta}{b}\left(1-e^{-b \omega}\right)\left(\frac{S^{*}}{1+\eta S^{*}}+\frac{\delta V^{*}}{1+\eta V^{*}}\right) I^{*}, \\
\beta e^{-b \tau}\left(\frac{S^{*}}{1+\eta S^{*}}+\frac{\delta V^{*}}{1+\eta V^{*}}\right) I^{*}-(\gamma+b+\alpha) I^{*}=0, \\
\gamma_{1} V^{*}-b R^{*}+\gamma\left(1-e^{-b \omega}\right) I^{*}=0 .
\end{gathered}
$$


The disease-free equilibrium point satisfies the constraints

$$
\begin{gathered}
E^{*}=I^{*}=0 \\
b\left(1-S^{*}\right)+v N^{*}\left(1-V_{c}\right)=0 \Longrightarrow S^{*}=1+\frac{v N^{*}\left(1-V_{c}\right)}{b}, \\
\gamma_{1} V^{*}-b R^{*}=0 \Longrightarrow V^{*}=\frac{b R^{*}}{\gamma_{1}} \\
-\left(\gamma_{1}+b\right) V^{*}+v N^{*} V_{c}=0 \Longrightarrow V^{*}=\frac{v N^{*} V_{c}}{\gamma_{1}+b}=\frac{b R^{*}}{\gamma_{1}} \\
N^{*}=S^{*}+V^{*}+R^{*}=1+\frac{v N^{*}\left(1-V_{c}\right)}{b}+\left(1+\frac{b}{\gamma_{1}}\right) R^{*} \\
=1+\frac{v N^{*}\left(1-V_{c}\right)}{b}+\frac{v N^{*} V_{c}}{b}=\frac{b+v N^{*}}{b} \Longrightarrow N^{*} \\
=\frac{b}{b-v} \quad \text { provided that } v<b .
\end{gathered}
$$

The proof follows directly from the above equations.

Remark 2.2. Note that if $\gamma_{1}=b$, then $R^{*}=V^{*}=\left(v V_{c} N^{*} / 2 b\right)=\left(v V_{c} /(2(b-v))\right)$. Note also that if $v=0$, as in the particular case of impulsive-free SVEIRS model obtained from that discussed in $[18,19]$, then the disease-free equilibrium satisfies $E^{*}=V^{*}=I^{*}=R^{*}=0, N^{*}=S^{*}=1$. In such a case, the model can be ran out with population normalized to unity.

Assertion 1. Assume that $\beta \leq(\gamma+b+\alpha) e^{b \tau}(b(1+\delta)-v) / b(1+\delta)$. Then,

$$
\begin{gathered}
\frac{S^{*}}{1+\eta S^{*}}+\frac{\delta V^{*}}{1+\eta V^{*}}=\frac{b-v V_{c}}{(1+\eta)(b-v)+\eta v\left(1-V_{c}\right)}+\frac{\delta v b V_{c}}{\left(\gamma_{1}+b\right)(b-v)+\eta v b V_{c}}, \\
\frac{S^{*}}{1+\eta S^{*}}+\frac{\delta V^{*}}{1+\eta V^{*}} \leq \frac{\left(\gamma+b+\alpha-\varepsilon_{\beta}\right) e^{b \tau}}{\beta},
\end{gathered}
$$

where $\mathbf{R}_{0+} \ni \varepsilon_{\beta}:=\gamma+b+\alpha-(\beta b(1+\delta) /(b(1+\eta)-v)) e^{-b \tau}$.

Proof. Note from Theorem 2.1 that the disease-free equilibrium point satisfies from simple direct calculations that

$$
\begin{aligned}
\frac{S^{*}}{1+\eta S^{*}}+\frac{\delta V^{*}}{1+\eta V^{*}} & =\frac{b-v V_{c}}{(1+\eta)(b-v)+\eta v\left(1-V_{c}\right)}+\frac{\delta v b V_{c}}{\left(\gamma_{1}+b\right)(b-v)+\eta v b V_{c}} \\
& =\frac{1}{S^{*-1}+\eta}+\frac{\delta}{V^{*-1}+\eta} \leq \frac{1+\delta}{N^{*-1}+\eta}=\frac{b(1+\delta)}{b(1+\eta)-v}=\frac{\left(\gamma+b+\alpha-\varepsilon_{\beta}\right) e^{b \tau}}{\beta}
\end{aligned}
$$


since $\min \left(S^{*^{-1}}, V^{*^{-1}}\right) \geq N^{*^{-1}}$ what also yields $\beta=\left(\gamma+b+\alpha-\varepsilon_{\beta}\right) e^{b \tau}((b(1+\eta)-v) / b(1+\delta))$, that is, $\varepsilon_{\beta}:=\gamma+b+\alpha-(\beta b(1+\delta) /(b(1+\eta)-v)) e^{-b \tau}$.

Note that the exposed population at the equilibrium defined by (1.3) can be equivalently described by a differential equation obtained by applying the Leibniz differentiation rule under the integral symbol to yield

$$
\dot{\tilde{E}}(t)=-b \tilde{E}(t)+\beta\left(\frac{S^{*}}{1+\eta S^{*}}+\frac{\delta V^{*}}{1+\eta V^{*}}\right)\left(\tilde{I}(t)-\widetilde{I}(t-\omega) e^{-b \omega}\right) .
$$

The local asymptotic stability of the disease-free equilibrium point is guaranteed by that of the linearized incremental system about it. The linearized model about the equilibrium becomes to be defined from (1.1), (1.2), (2.10) and (1.4), (1.5) by the state vector $\tilde{x}(t):=$ $(\widetilde{S}(t), \tilde{V}(t), \widetilde{E}(t), \widetilde{I}(t), \widetilde{R}(t))^{T}$ which satisfies the differential system

$$
\dot{\tilde{x}}(t)=A_{0}^{*} \tilde{x}(t)+A_{\tau}^{*} \tilde{x}(t-\tau)+A_{\omega}^{*} \tilde{x}(t-\omega) ; \quad \tilde{x}(0)=\tilde{x}_{0},
$$

where, after using the identities in Theorem 2.1 related to the equilibrium point and provided that Assertion 1 holds, one gets

$$
\begin{aligned}
& A_{0}^{*}=A_{0 d}^{*}+\tilde{A}_{0}^{*} \text {, } \\
& :=\left[\begin{array}{ccccc}
v\left(1-V_{c}\right)-b & v\left(1-V_{c}\right) & v\left(1-V_{c}\right) & v\left(1-V_{c}\right)-\frac{\beta S^{*}}{1+\eta S^{*}} & v\left(1-V_{c}\right) \\
v V_{c} & v V_{c}-\left(\gamma_{1}+b\right) & v V_{c} & v V_{c}-\frac{\delta \beta V^{*}}{1+\eta V^{*}} & v V_{c} \\
0 & 0 & -b & \beta\left(\frac{S^{*}}{1+\eta S^{*}}+\frac{\delta V^{*}}{1+\eta V^{*}}\right) & 0 \\
0 & 0 & 0 & -(\gamma+b+\alpha) & 0 \\
0 & \gamma_{1} & 0 & \gamma & -b
\end{array}\right], \\
& :=\left[\begin{array}{ccccc}
v\left(1-V_{c}\right)-b & v\left(1-V_{c}\right) & v\left(1-V_{c}\right) & v\left(1-V_{c}\right)-\frac{\beta\left(b+v\left(1-V_{c}\right) N^{*}\right)}{b+\eta\left(b+v\left(1-V_{c}\right) N^{*}\right)} & v\left(1-V_{c}\right) \\
v V_{c} & v V_{c}-\left(\gamma_{1}+b\right) & v V_{c} & v V_{c}-\frac{\delta \beta v V_{c} N^{*}}{\gamma_{1}+b+\eta v V_{c} N^{*}} & v V_{c} \\
0 & 0 & -b & \left(\gamma+b+\alpha-\varepsilon_{\beta^{\prime}}\right) e^{b \tau} & 0 \\
0 & 0 & 0 & -(\gamma+b+\alpha) & 0 \\
0 & \gamma_{1} & 0 & \gamma & -b
\end{array}\right],
\end{aligned}
$$


where $\varepsilon_{\beta^{\prime}}>\varepsilon_{\beta}$ is a real constant, and

$$
\begin{gathered}
A_{0 d}^{*}:=\operatorname{Diag}\left(v\left(1-V_{c}\right)-b, v V_{c}-\left(\gamma_{1}+b\right),-b,-(\gamma+b+\alpha),-b\right), \\
\tilde{A}_{0}^{*}:=\left[\begin{array}{ccccc}
0 & v\left(1-V_{c}\right) & v\left(1-V_{c}\right) & v\left(1-V_{c}\right)-\frac{\beta\left(b+v\left(1-V_{c}\right) N^{*}\right)}{b+\eta\left(b+v\left(1-V_{c}\right) N^{*}\right)} & v\left(1-V_{c}\right) \\
v & 0 & v V_{c} & v V_{c}-\frac{\delta \beta v V_{c} N^{*}}{\gamma_{1}+b+\eta v V_{c} N^{*}} & v V_{c} \\
0 & 0 & 0 & \left(\gamma+b+\alpha-\varepsilon_{\beta^{\prime}}\right) e^{b \tau} & 0 \\
0 & 0 & 0 & 0 & 0 \\
0 & \gamma_{1} & 0 & \gamma & 0
\end{array}\right],
\end{gathered}
$$

and the matrices $A_{\tau}^{*}$ and $A_{\omega}^{*}$ are entry-wise defined by

$$
\begin{gathered}
\left(A_{\tau}^{*}\right)_{44}=\gamma+b+\alpha-\varepsilon_{\beta^{\prime}}, \quad\left(A_{\omega}^{*}\right)_{14}=\gamma e^{-b \omega}, \\
\left(A_{\omega}^{*}\right)_{34}=-\left(\gamma+b+\alpha-\varepsilon_{\beta^{\prime}}\right) e^{b(\tau-\omega)}, \quad\left(A_{\omega}^{*}\right)_{54}=-\gamma e^{-b \omega},
\end{gathered}
$$

with all the remaining entries being zero. The following inequalities apply for equivalent norms of either vectors or vector-induced norms of matrices $M$ of dimension or, respectively, order $n$ :

$$
n^{-1}\|M\|_{2} \leq n^{-1 / 2}\|M\|_{\infty} \leq\|M\|_{2} \leq n^{1 / 2}\|M\|_{1} \leq n\|M\|_{2}
$$

Thus, one gets from the above inequalities (2.16) that

$$
\left\|A_{\tau}^{*}\right\|_{2}+\left\|A_{\omega}^{*}\right\|_{2} \leq \sqrt{5}\left(\left\|A_{\tau}^{*}\right\|_{\infty}+\left\|A_{\omega}^{*}\right\|_{\infty}\right) \leq \sqrt{5}(\gamma+b+\alpha) \max \left(1, e^{b(\tau-\omega)}\right) \leq \bar{\gamma},
$$

where

$$
\bar{\gamma}= \begin{cases}\sqrt{5}(\gamma+b+\alpha), & \text { if } \tau \leq \omega \\ \sqrt{5}(\gamma+b+\alpha) e^{b(\tau-\omega)}, & \text { if } \tau>\omega .\end{cases}
$$

Note from (2.18) that $\sqrt{5}(\gamma+b+\alpha) e^{b(\tau-\omega)} \leq b-b_{0}$ for a given $b$ and any given positive real constant $b_{0}<b$ if $(\gamma+b+\alpha)$ and $(\tau-\omega)$, if positive, are small enough such that, equivalently,

$$
-\infty \leq \frac{1}{2} \ln 5+\ln (\gamma+b+\alpha)+b(\tau-\omega) \leq \ln \left(b-b_{0}\right) .
$$


Thus, one gets from (2.17)-(2.19) that

$$
\left\|A_{\tau}^{*}\right\|_{2}+\left\|A_{\omega}^{*}\right\|_{2} \leq \bar{\gamma} \leq b-b_{0}
$$

On the other hand, we can use from L'Hopital rule the following limit relations in the entries $(1,4)$ and $(2,4)$ of the matrix $\tilde{A}_{0}^{*}$ :

$$
\frac{\beta\left(b+v\left(1-V_{c}\right) N^{*}\right)}{b+\eta\left(b+v\left(1-V_{c}\right) N^{*}\right)} \longrightarrow \frac{\beta}{1+\eta} ; \quad \frac{\delta \beta v V_{c} N^{*}}{\gamma_{1}+b+\eta v V_{c} N^{*}} \longrightarrow 0 \quad \text { as } b \longrightarrow \infty
$$

if the remaining parameters remain finite and then $N^{*}=S^{*}=1$ and $E^{*}=I^{*}=V^{*}=R^{*}=0$ from Theorem 2.1. By continuity with respect to parameters, for any sufficiently large $M \in$ $\mathbf{R}_{0+}$, there exist $\varepsilon_{1,2}=\varepsilon_{1,2}(M) \in \mathbf{R}_{0+}$ with $\varepsilon_{1,2} \rightarrow 0$ as $t \rightarrow \infty$ such that for $b \geq M$,

$$
\frac{\beta\left(b+v\left(1-V_{c}\right) N^{*}\right)}{b+\eta\left(b+v\left(1-V_{c}\right) N^{*}\right)} \leq \frac{\beta+\varepsilon_{1}}{1+\eta} ; \quad \frac{\delta \beta v V_{c} N^{*}}{\gamma_{1}+b+\eta v V_{c} N^{*}} \leq \varepsilon_{2}
$$

and, one gets from (2.14),

$$
\left|\tilde{A}_{0}^{*}\right|=\left[\begin{array}{ccccc}
0 & v\left(1-V_{c}\right) & v\left(1-V_{c}\right) & \left|v\left(1-V_{c}\right)-\frac{\beta+\varepsilon_{1}}{1+\eta}\right| & v\left(1-V_{c}\right) \\
v V_{c} & 0 & v V_{c} & \left|v V_{c}-\varepsilon_{2}\right| & v V_{c} \\
0 & 0 & 0 & \left(\gamma+b+\alpha-\varepsilon_{\beta^{\prime}}\right) e^{b \tau} & 0 \\
0 & 0 & 0 & 0 & 0 \\
0 & \gamma_{1} & 0 & \gamma & 0
\end{array}\right],
$$

and for $b$ being large enough such that it satisfies

$$
b \geq \max \left(\frac{1}{\tau} \max \left(\ln \frac{\gamma+\gamma_{1}}{\gamma+b+\alpha}, \ln \frac{4 \max (1, v)}{\gamma+b+\alpha}\right), b_{a}\right),
$$

with $b_{a}$ being some existing real positive constant, depending on the vaccination constant $V_{c}$, such that $v\left(1-V_{c}\right) \geq\left(\left(\beta+\varepsilon_{1}\right) /(1+\eta)\right)$, it follows from inspection of $(2.22),(2.23)$ that $\left\|\tilde{A}_{0}^{*}\right\|_{\infty} \leq$ $(\gamma+b+\alpha) e^{b \tau}$. Using again (2.16), (2.17), it follows that the following close constraint to (2.19) for large enough $b$ :

$-\infty \leq \frac{1}{2} \ln 5+\ln (\gamma+b+\alpha)+b(\tau-\omega) \leq \frac{1}{2} \ln 5+\ln (\gamma+b+\alpha)+b \tau+\ln \left(1+e^{-b \omega}\right) \leq \ln \left(b-b_{0}\right)$ 
guarantees

$$
\begin{aligned}
\left\|A_{\tau}^{*}\right\|_{2}+\left\|A_{\omega}^{*}\right\|_{2}+\left\|\tilde{A}_{0}^{*}\right\|_{2} & \leq \sqrt{5}\left(\left\|A_{\tau}^{*}\right\|_{\infty}+\left\|A_{\omega}^{*}\right\|_{\infty}+\left\|\tilde{A}_{0}^{*}\right\|_{\infty}\right) \\
& \leq \sqrt{5}(\gamma+b+\alpha)\left(\max \left(1, e^{b(\tau-\omega)}\right)+e^{b \tau}\right) \leq \bar{\gamma}_{1},
\end{aligned}
$$

where

$$
\bar{\gamma}_{1}(>\bar{\gamma})= \begin{cases}\sqrt{5}(\gamma+b+\alpha)\left(1+e^{b \tau}\right), & \text { if } \tau \leq \omega \\ \sqrt{5}(\gamma+b+\alpha)\left(e^{b \tau}\left(1+e^{-b \omega}\right)\right), & \text { if } \tau>\omega\end{cases}
$$

On the other hand, note that the linearized system (2.11)-(2.17) is asymptotically stable if and only if

$$
\operatorname{det}\left(s I-A_{0 d}^{*}-\widetilde{A}_{0}^{*}-\widetilde{A}_{\tau}^{*} e^{-\tau s}-\widetilde{A}_{\omega}^{*} e^{-\omega s}\right) \neq 0 ; \quad \forall s \in \mathbf{C}_{0+}:=\{s \in \mathbf{C}: \operatorname{Re} s \geq 0\}
$$

which is guaranteed under the two conditions below:

(1) $\operatorname{det}\left(s I-A_{0 d}^{*}\right) \neq 0$, for all $s \in \mathbf{C}_{0+}:=\{s \in \mathbf{C}: \operatorname{Re} s \geq 0\}$, equivalently, $A_{0 d}^{*}$ is a stability matrix

(2) the $\ell_{2}$-matrix measure $\mu_{2}\left(A_{0 d}^{*}\right)$ of $\left(A_{0 d}^{*}\right)$ is negative, and, furthermore, the following constraint holds

$$
\bar{\gamma}_{1} \leq b-\max \left(\left|\gamma_{1}-v V_{c}\right|, v\left(1-V_{c}\right)\right)
$$

which guarantees the above stability Condition 2 via (2.26), (2.27), and (2.13)

$$
\begin{aligned}
\left\|\tilde{A}_{0}^{*}\right\|_{2}+\left\|\tilde{A}_{\tau}^{*}\right\|_{2}+\left\|\tilde{A}_{\omega}^{*}\right\|_{2} & \leq \sqrt{5}(\gamma+b+\alpha)\left(\max \left(1, e^{b(\tau-\omega)}\right)+e^{b \tau}\right) \leq \bar{\gamma}_{1} \\
& <\left|\mu_{2}\left(A_{0 d}^{*}\right)\right|=\frac{1}{2}\left|\lambda_{\max }\left(A_{0 d}^{*}+A_{0 d}^{*^{T}}\right)\right|=\left|\lambda_{\max }\left(A_{0 d}^{*}\right)\right| \\
& =b-\max \left(\left|\gamma_{1}-v V_{c}\right|, v\left(1-V_{c}\right)\right) .
\end{aligned}
$$

The following result is proven from Theorem 2.1, by taking into account the above asymptotic stability conditions for the linearized incremental system about the disease-free equilibrium point, which imply that of the nonlinear one (1.1)-(1.5) about the equilibrium point, and the related former discussion.

Theorem 2.3. Assume that $\beta \leq(\gamma+b+\alpha) e^{b \tau}((b(1+\eta)-v) / b(1+\delta))$. Then, there is a sufficiently large $b>\max \left(\left|\gamma_{1}-v V_{c}\right|, v\left(1-V_{c}\right)\right)$ such that the disease-free equilibrium point is locally asymptotically stable for any constant vaccination $V_{c} \in[0,1]$ and a sufficiently small amount $(\gamma+b+\alpha)$, a sufficiently small delay $\tau$ and a sufficiently small difference delay $(\tau-\omega)$ (this being applicable if $\tau>\omega$ ) such that (2.30) holds. 
Note that the statement of Theorem 2.3 guarantees the local stability of the disease-free equilibrium point under its existence condition of Theorem 2.1 requiring $v<b$.

\section{The Existence of Endemic Equilibrium Points, Uniqueness Issues, and Some Related Characterizations}

The existence of endemic equilibrium points which keep alive the disease propagation is now discussed. It is proven that there is a unique equilibrium point with physical meaning since all the partial populations are nonnegative.

Theorem 3.1. Assume that $\omega>0$. Then, the following properties hold.

(i) Assume that $\beta \geq\left(\left(\eta e^{b \tau}(\gamma+b+\alpha)\right) /(1+\delta)\right)$ for $V_{c}>0$ and $\beta \geq \eta e^{b \tau}(\gamma+b+\alpha)$ for $V_{c}=0$. Thus, there is at least one endemic equilibrium point at which the susceptible, vaccinated, infected, exposed, and recovered populations are positive and the vaccinated population is zero if and only if $V_{c}=0$ (i.e., in the absence of vaccination action). Furthermore, such an equilibrium point satisfies the constraints

$$
\begin{gathered}
E^{*}=\frac{\beta}{b}\left(1-e^{-b \omega}\right)\left(\frac{S^{*}}{1+\eta S^{*}}+\frac{\delta V^{*}}{1+\eta V^{*}}\right) I^{*}>0 \\
\min \left(S^{*}+\delta V^{*}, \frac{1+\delta}{\eta}\right) \geq \frac{S^{*}}{1+\eta S^{*}}+\frac{\delta V^{*}}{1+\eta V^{*}}=\frac{e^{b \tau}(\gamma+b+\alpha)}{\beta}>0 \\
R^{*}=\frac{\gamma_{1} V^{*}+\gamma\left(1-e^{-b \omega}\right) I^{*}}{b} \geq \frac{\gamma\left(1-e^{-b \omega}\right) I^{*}}{b}>0 .
\end{gathered}
$$

(ii) If the disease transmission constant is small enough satisfying $\beta<\bar{\beta}:=\left(\eta e^{b \tau}(\gamma+b+\right.$ $\alpha) /(1+\delta))$ for $V_{c}>0$, and $\beta<\eta e^{b \tau}(\gamma+b+\alpha)$ for $V_{c}=0$, then there is no reachable endemic equilibrium point.

Proof. The endemic equilibrium point is calculated as follows:

$$
\begin{aligned}
& \beta e^{-b \tau}\left(\frac{S^{*}}{1+\eta S^{*}}+\frac{\delta V^{*}}{1+\eta V^{*}}\right)-(\gamma+b+\alpha)=0, \\
& E^{*}=\frac{\beta}{b}\left(1-e^{-b \omega}\right)\left(\frac{S^{*}}{1+\eta S^{*}}+\frac{\delta V^{*}}{1+\eta V^{*}}\right) I^{*}>0
\end{aligned}
$$

with

$$
\begin{gathered}
E^{*}>0, \quad I^{*}>0, \\
\frac{S^{*}}{1+\eta S^{*}}+\frac{\delta V^{*}}{1+\eta V^{*}}=\frac{e^{b \tau}(\gamma+b+\alpha)}{\beta}>0
\end{gathered}
$$

(since, otherwise, the above disease-free equilibrium point would be considered). 
$S^{*}>0$ since, otherwise, the following contradiction would follow:

$$
0<b+\gamma I^{*} e^{-b \omega}+v N^{*}\left(1-V_{c}\right)=0 .
$$

$V^{*}=0$ if and only if $V_{c}=0$, since otherwise for $V_{c}>0$ and $V^{*}=0$, it would follow that $v N^{*} V_{c}=0$, what is only possible in the disease-free equilibrium if the total population is extinguished, is a contradiction at the endemic point

$$
R^{*}=\frac{r_{1} V^{*}+\gamma\left(1-e^{-b \omega}\right) I^{*}}{b} \geq \frac{\gamma\left(1-e^{-b \omega}\right) I^{*}}{b}>0, \text { for } \omega \neq 0
$$

Remark 3.2. Note that if $\omega=0$, then it follows from (1.3) and (2.4) that $E(t)=E^{*}=0$, for all $t \in$ $\mathbf{R}_{0+}$ so that the SVEIRS model (1.1)-(1.5) becomes a simpler SVIRS one without specification of the exposed population dynamics.

Remark 3.3. Note that, under the constraints in Theorem 3.1(ii) for $\beta$, if there is no reachable endemic equilibrium point because $\beta<\bar{\beta}$, then the solution trajectory of (1.1)-(1.5) can only either converge to the disease-free equilibrium point provided that it is at least locally asymptotically stable or to be bounded converging or not to an oscillatory solution or to diverge to an unbounded total population depending on the values of the parameterization of the model (1.1)-(1.5). Note that the endemic-free disease transmission constant upperbound $\bar{\beta}$ increases as $\eta, \tau$ and $(\gamma+b+\alpha)$ increase and also as $\delta$ decreases.

If $V_{c}>0$, then it follows from Theorem 3.1 that there exist positive constants $\alpha_{S}, \alpha_{V}$ $\alpha_{E}, \alpha_{I}$, and $\alpha_{R}$ satisfying $\alpha_{S}^{-1}+\alpha_{V}^{-1}+\alpha_{E}^{-1}+\alpha_{I}^{-1}+\alpha_{R}^{-1}=1$ such that the endemic equilibrium points, if any, satisfy the constraints

$$
N^{*}=\alpha_{S} S^{*}=\alpha_{V} V^{*}=\alpha_{E} E^{*}=\alpha_{I} I^{*}=\alpha_{R} R^{*}
$$

so that one gets from (3.3)-(3.7) that

$$
\begin{gathered}
R^{*}=\frac{\gamma_{1} / \alpha_{V}+\gamma\left(1-e^{-b \omega}\right) / \alpha_{I}}{b} \alpha_{R} R^{*}=\frac{\gamma_{1} \alpha_{I}+\gamma\left(1-e^{-b \omega}\right) \alpha_{V}}{b \alpha_{I} \alpha_{V}} \alpha_{R} R^{*} \\
\frac{\beta}{b}\left(1-e^{-b \omega}\right) \frac{1+\delta}{1+\eta} \leq \frac{E^{*}}{I^{*}}=\frac{\alpha_{I}}{\alpha_{E}}=\frac{\beta}{b}\left(1-e^{-b \omega}\right)\left(\frac{S^{*}}{1+\eta S^{*}}+\frac{\delta V^{*}}{1+\eta V^{*}}\right) \leq \frac{\beta}{b}\left(1-e^{-b \omega}\right) \frac{1+\delta}{\eta}
\end{gathered}
$$

if $\min \left(S^{*}, V^{*}\right) \geq 1$, otherwise, then only the upper-bounding constraint holds in (3.10)

$$
\begin{gathered}
b-\left(b+\frac{\beta \alpha_{S} S^{*}}{\alpha_{I}\left(1+\eta S^{*}\right)}\right) S^{*}+\gamma \frac{\alpha_{S}}{\alpha_{I}} S^{*} e^{-b \omega}+v \alpha_{S} S^{*}\left(1-V_{c}\right)=0, \\
\frac{\alpha_{V} V^{*}}{\alpha_{S}+\alpha_{V} \eta V^{*}}+\frac{\delta V^{*}}{1+\eta V^{*}}=\frac{e^{b \tau}(\gamma+b+\alpha)}{\beta} .
\end{gathered}
$$


Equation (3.9) is equivalent, since $R^{*}>0$ at the endemic equilibrium point, to

$$
\frac{\gamma_{1} \alpha_{I} \alpha_{R}+\gamma\left(1-e^{-b \omega}\right) \alpha_{V} \alpha_{R}}{b \alpha_{I} \alpha_{V}}=1
$$

Equation (3.11) is equivalent to

$$
\begin{aligned}
& {\left[\alpha_{S} \eta\left(v \alpha_{I}\left(1-V_{c}\right)+\gamma e^{-b \omega}\right)+\beta \alpha_{S}-b \alpha_{I} \eta\right] S^{*^{2}}} \\
& \quad+\left[\alpha_{S}\left(\gamma e^{-b \omega}+v \alpha_{I}\left(1-V_{c}\right)\right)+b \alpha_{I}(\eta-1)\right] S^{*}+b \alpha_{I}=0 .
\end{aligned}
$$

Equation (3.14) is an algebraic equation of real coefficients of the form $a S^{*^{2}}+d S^{*}+c=0$ with $c>0$. Such an equation has two positive real roots if $a>0, d<0$ and $d^{2} \geq 4 a c$ and one positive real root if $a<0$ and $d>0$. Thus, since there is a nonzero susceptible population at an endemic equilibrium point, then either (3.15) below holds

$$
\begin{gathered}
\alpha_{S} \eta\left(v \alpha_{I}\left(1-V_{c}\right)+\gamma e^{-b \omega}\right)+\beta \alpha_{S}>b \alpha_{I} \eta \\
\alpha_{S}\left(\gamma e^{-b \omega}+v \alpha_{I}\left(1-V_{c}\right)\right)<b \alpha_{I}(1-\eta), \quad \text { provided that } \eta<1 \\
{\left[\alpha_{S}\left(\gamma e^{-b \omega}+v \alpha_{I}\left(1-V_{c}\right)\right)+b \alpha_{I}(\eta-1)\right]^{2} \geq 4 b \alpha_{I}\left[\alpha_{S} \eta\left(v \alpha_{I}\left(1-V_{c}\right)+\gamma e^{-b \omega}\right)+\beta \alpha_{S}-b \alpha_{I} \eta\right]}
\end{gathered}
$$

or, alternatively,

$$
\begin{gathered}
\beta<\frac{\alpha_{I}}{\alpha_{S}} b \eta-\left(v \alpha_{I}\left(1-V_{c}\right)+\gamma e^{-b \omega}\right) \eta=\frac{\eta}{I^{*}}\left[b S^{*}-\left(v N^{*}\left(1-V_{c}\right)+\gamma e^{-b \omega}\right)\right], \\
b<\frac{\alpha_{S}\left(\gamma e^{-b \omega}+v \alpha_{I}\left(1-V_{c}\right)\right)}{\alpha_{I}(1-\eta)}=\frac{\gamma e^{-b \omega} I^{*}+v N^{*}\left(1-V_{c}\right)}{S^{*}(1-\eta)},
\end{gathered}
$$

with $\eta<1$ hold. On the other hand, (3.12) is equivalent to

$$
\alpha_{V} \beta_{0}\left(1+\eta V^{*}\right) V^{*}+\delta \beta_{0} V^{*}\left(\alpha_{S}+\eta \alpha_{V} V^{*}\right)=\left(1+\eta V^{*}\right)\left(\alpha_{S}+\eta \alpha_{V} V^{*}\right)
$$

where $\beta_{0}:=\beta / e^{b \tau}(\gamma+b+\alpha)$ so that (3.17) is of the form specifically as follows:

$$
a V^{*^{2}}+d V^{*}+c \equiv\left(\eta-(1+\delta) \beta_{0}\right) \alpha_{V} \eta V^{*^{2}}+\left(\alpha_{V}\left(\eta-\beta_{0}\right)+\left(\eta-\delta \beta_{0}\right) \alpha_{S}\right) V^{*}+\alpha_{S}=0
$$


Now, the same reasoning as that used for the susceptible endemic equilibrium component is applied to (3.18) to conclude that, since there is a nonzero vaccinated population for at most two endemic equilibrium points with $\min \left(S^{*}, V^{*}\right) \geq 1$, then

$$
(\gamma+b+\alpha) e^{b \tau} \frac{\eta}{1+\delta} \leq \beta \leq(\gamma+b+\alpha) e^{b \tau} \frac{1+\eta}{1+\delta}
$$

which is obtained from (3.2), and either

$$
\begin{gathered}
\alpha_{V}\left(\beta_{0}-\eta\right)+\left(\delta \beta_{0}-\eta\right) \alpha_{S}=\left(\alpha_{V}+\alpha_{S} \delta\right) \beta_{0}-\eta \alpha_{V}-\eta \alpha_{S}>0, \\
\beta_{0}>\frac{\eta \alpha_{V}+\eta \alpha_{S}}{\alpha_{V}+\alpha_{S} \delta} \Longleftrightarrow \beta>\frac{\eta \alpha_{V}+\eta \alpha_{S}}{\alpha_{V}+\alpha_{S} \delta} e^{b \tau}(\gamma+b+\alpha), \\
\left(\alpha_{V}\left(\eta-\beta_{0}\right)+\left(\eta-\delta \beta_{0}\right) \alpha_{S}\right)^{2}>4\left(\eta-(1+\delta) \beta_{0}\right) \eta \alpha_{V} \alpha_{S}
\end{gathered}
$$

or, $a<0, d>0$ in (3.18). The uniqueness of the endemic equilibrium point with all partial populations being nonnegative for all time is now proven as follows. First, define auxiliary variables

$$
A=\eta\left(v \alpha_{I}\left(1-V_{c}\right)+\gamma e^{-b \omega}\right)>0 ; \quad B=\frac{A}{\eta}=v \alpha_{I}\left(1-V_{c}\right)+\gamma e^{-b \omega}>0 .
$$

Thus, since $N^{*}=\alpha_{S} S^{*},(3.14)$ can be rewritten as follows:

$$
-b \alpha_{I} \eta S^{*^{2}}+\left[b \alpha_{I}(\eta-1)+N^{*}(A+\beta)\right] S^{*}+\left(b \alpha_{I}+N^{*} B\right)=0 .
$$

If such an equation has two positive real roots for the susceptible equilibrium (implicitly depending on $\left.N^{*}\right)$, then either $b \alpha_{I} \eta<0$ or $\left(b \alpha_{I}+N^{*} B\right)<0$ what is impossible and leads to a contradiction. Then, there is a unique nonnegative susceptible population $S^{*} \geq 0$ at the two potentially existing endemic equilibrium points provided that the total population $N^{*}$ at the endemic equilibrium point is unique. Furthermore, simple inspection of the above equation implies strict positivity $S^{*}>0$. On the other hand, it follows from Theorem 3.1, (3.5), that $\delta V^{*} /\left(1+\eta V^{*}\right)=\left(e^{b \tau}(\gamma+b+\alpha) / \beta\right)-\left(S^{*} /\left(1+\eta S^{*}\right)\right)$, which has a unique solution in $V^{*}$ for a given $S^{*}$. Since there is a unique $S^{*}>0$, then there is a unique $V^{*}>0$ as a result. From (2.5) in the proof of Theorem 2.1, there is also a unique population at the infected population endemic equilibrium $I^{*}>0$, then unique related exposed and recovered equilibrium populations $E^{*}>$ 0 and $R^{*}>0$ from (2.4) and (2.6), respectively. Thus, there is a unique endemic equilibrium point with all the partial populations being nonnegative. The above discussion concerning the existence of a unique endemic equilibrium point with all the partial populations being nonnegative is summarized as follows.

Theorem 3.4. Assume that $V_{c} \in(0,1]$ and that $(\gamma+b+\alpha) e^{b \tau}(\eta /(1+\delta)) \leq \beta \leq(\gamma+b+\alpha) e^{b \tau}((1+$ $\eta) /(1+\delta)$ ) (the upper-bounding condition does not hold if $\left.\min \left(S^{*}, V^{*}\right)<1\right)$ so that $N^{*}=\alpha_{S} S^{*}=$ $\alpha_{V} V^{*}=\alpha_{E} E^{*}=\alpha_{I} I^{*}=\alpha_{R} R^{*}$ for some positive constants $\alpha_{S}, \alpha_{V}, \alpha_{E}, \alpha_{I}$ and $\alpha_{R}$. If $N^{*}$ is unique at 
the endemic equilibrium then, there is a unique endemic equilibrium point with all the corresponding partial populations being positive, and the following parametrical constraints hold:

$$
\begin{gathered}
\alpha_{S}^{-1}+\alpha_{V}^{-1}+\alpha_{E}^{-1}+\alpha_{I}^{-1}+\alpha_{R}^{-1}=1 \\
\frac{\beta}{b}\left(1-e^{-b \omega}\right) \frac{1+\delta}{1+\eta} \leq \frac{\alpha_{I}}{\alpha_{E}} \leq \frac{\beta}{b}\left(1-e^{-b \omega}\right) \frac{1+\delta}{\eta} .
\end{gathered}
$$

The constants $\alpha_{S}, \alpha_{I}$, and $\alpha_{V}$ satisfy either (3.15), or (3.16), and the constraint $\alpha_{V}\left(\eta-\beta_{0}\right)+(\eta-$ $\left.\delta \beta_{0}\right) \alpha_{S}>0$ so that $d>0$ in (3.18).

This result will be combined with some issues concerning the existence of limits of all the partial population at infinite time to conclude that there is a unique total population at the endemic equilibrium points so that, from Theorem 3.4, there is a unique endemic equilibrium point (see Remark 5.1 and Theorem 5.2 in Section 5).

\section{About Infection Propagation and the Properties of Uniform Boundedness of the Total Population and Positivity of All the Partial Populations}

This section discuses briefly the monotone increase of the infected population and the boundedness of the total population as well as the positivity of the model.

Theorem 4.1. If the infection propagates through $(t-\tau, t)$ with the infected population being monotone increasing, then

$$
\frac{S(\sigma)}{1+\eta S(\sigma)}+\frac{\delta V(\sigma)}{1+\eta V(\sigma)} \geq \frac{\gamma+b+\alpha}{\beta} e^{b \sigma} ; \quad \forall \sigma \in\left(t^{*}-2 \tau, t^{*}-\tau\right)
$$

Proof. Note from (1.4) that for $t \in\left(t^{*}-2 \tau, t^{*}\right)$

$$
\dot{I}(t)>0 \Longleftrightarrow \frac{I(t)}{I(t-\tau)}<\frac{\beta e^{-b \tau}}{\gamma+b+\alpha}\left(\frac{S(t-\tau)}{1+\eta S(t-\tau)}+\frac{\delta V(t-\tau)}{1+\eta V(t-\tau)}\right)
$$

and if, furthermore, $I(t)>I(t-\tau)$ for $t \in\left(t^{*}-\tau, t^{*}\right)$, then

$$
1<\frac{I(t)}{I(t-\tau)}<\frac{\beta e^{-b \tau}}{\gamma+b+\alpha}\left(\frac{S(t-\tau)}{1+\eta S(t-\tau)}+\frac{\delta V(t-\tau)}{1+\eta V(t-\tau)}\right)
$$

Now, rewrite (1.3) in differential equivalent form by using Leibnitz's rule as follows:

$$
\begin{aligned}
\dot{E}(t)= & -b E(t) \\
& +\beta\left[\left(\frac{S(t)}{1+\eta S(t)}+\frac{\delta V(t)}{1+\eta V(t)}\right) I(t)-\left(\frac{S(t-\omega)}{1+\eta S(t-\omega)}+\frac{\delta V(t-\omega)}{1+\eta V(t-\omega)}\right) I(t-\omega) e^{-b \omega}\right] .
\end{aligned}
$$


Theorem 4.2. Assume that $v<b$. Then, the following properties hold provided that the SVEIR epidemic model (1.1)-(1.5) has nonnegative solution trajectories of all the partial populations for all time:

(i) assume furthermore that $\psi:=\left(e^{v \tau}+\left(\beta(1+\delta)\left(1-e^{-(b-v) \tau}\right) / \eta(b-v)\right)\right) e^{-b \tau}<1$, then the total population is uniformly bounded for all time, irrespective of the susceptible and vaccinated populations, for any bounded initial conditions and

$$
\limsup _{t \rightarrow \infty} N(t) \leq \frac{1-e^{-(b-v) \tau}}{b-v}(1-\psi)^{-1}<\infty
$$

(ii) assume that the disease transmission constant is large enough satisfying $\beta \geq(1 /(1+$ $\delta) \sup _{t \in \mathbf{R}_{0+}}\left(b \eta(1+\eta) /\left(\eta e^{-b \omega} I(t-\omega)-(1+\eta) e^{-b \tau} I(t-\tau)\right)\right)$ subject to $(\eta /(1+\eta))>$ $e^{b(\omega-\tau)}$ and $\omega<\tau$, then $N: \mathbf{R}_{0+} \rightarrow \mathbf{R}_{0+}$ is monotone decreasing and of negative exponential order so that the total population exponentially extinguishes as a result.

Proof. Consider the SVEIRS model in differential form described by (1.1), (1.2), (1.4), (1.5), and (4.4). Summing up the five equations, one gets directly

$$
\begin{aligned}
\dot{N}(t)= & (v-b) N(t)+b-\alpha I(t) \\
+ & \beta\left[\left(\frac{S(t-\tau) I(t-\tau)}{1+\eta S(t-\tau)}+\frac{\delta V(t-\tau) I(t-\tau)}{1+\eta V(t-\tau)}\right) e^{-b \tau}\right. \\
& \left.\quad-\left(\frac{S(t-\omega) I(t-\omega)}{1+\eta S(t-\omega)}+\frac{\delta V(t-\omega) I(t-\omega)}{1+\eta V(t-\omega)}\right) e^{-b \omega}\right] \\
\leq & (v-b) N(t)+b+\beta\left(\frac{S(t-\tau)}{1+\eta S(t-\tau)}+\frac{\delta V(t-\tau)}{1+\eta V(t-\tau)}\right) I(t-\tau) e^{-b \tau} \\
\leq & (v-b) N(t)+b+\beta \frac{1+\delta}{\eta} e^{-b \tau} I(t-\tau) \leq(v-b) N(t)+b+\beta \frac{1+\delta}{\eta} e^{-b \tau} N(t-\tau)
\end{aligned}
$$

since $S(t) /(1+\eta S(t))+\delta V(t) /(1+\eta V(t)) \leq(1+\delta) / \eta$; for all $t \in \mathbf{R}_{0+}$. Then,

$$
N(t) \leq \psi \sup _{t-\tau \leq \sigma \leq t} N(\sigma)+\frac{b\left(1-e^{-(b-v) \tau}\right)}{b-v}<\infty ; \quad \forall t \in \mathbf{R}_{0+},
$$

and Property (i) follows since $\psi<1$. Two cases are now discussed separately related to the proof of Property (ii).

(a) Note that if the solution trajectory is positive subject to $\min (S(t), V(t)) \geq 1$ (equivalently if $\max \left(S^{-1}(t), V^{-1}(t)\right) \leq 1$ ), then

$$
0<\frac{1+\delta}{1+\eta} \leq \frac{S(t)}{1+\eta S(t)}+\frac{\delta V(t)}{1+\eta V(t)} \leq \frac{1+\delta}{\eta}
$$


so that one gets from (4.6)

$$
\begin{aligned}
\dot{N}(t) & \leq(v-b) N(t)-\alpha I(t)+\left(b-\beta\left[\frac{1+\delta}{1+\eta} I(t-\omega) e^{-b \omega}-\frac{1+\delta}{\eta} I(t-\tau) e^{-b \tau}\right]\right) \\
& \leq-(b-v) N(t)-\alpha I(t) \leq-(b-v) N(t) \leq 0
\end{aligned}
$$

with identically zero upper-bound in (4.10) holds for some $t \in \mathbf{R}_{0+}$ if and only if $N(t)=I(t)=$ 0 since $b>v$ and

$$
\beta \geq \frac{1}{1+\delta}\left(\frac{b \eta(1+\eta)}{\eta e^{-b \omega} I(t-\omega)-(1+\eta) I(t-\tau) e^{-b \tau}}\right)>0
$$

provided that $(\eta /(1+\eta)) e^{b(\omega-\tau)}$ with $\omega<\tau$. Then, $N(t) \leq e^{-(b-v) t} N(0)<N\left(t^{\prime}\right)$, for all $t, t^{\prime}(<t) \in \mathbf{R}_{0+}$.

(b) If $\max (S(t), V(t)) \leq 1$ (equivalently, if $\min \left(S^{-1}(t), V^{-1}(t)\right) \geq 1$ ), then

$$
0 \leq \frac{S(t)}{1+\eta S(t)}+\frac{\delta V(t)}{1+\eta V(t)} \leq \frac{1+\delta}{1+\eta} \leq \frac{1+\delta}{\eta}
$$

so that (4.10) still holds and the same conclusion arises. Thus, Property (ii) is proven.

A brief discussion about positivity is summarized in the next result.

Theorem 4.3. Assume that $V_{c} \in[0,1]$. Then, the SVEIRS epidemic model (1.1)-(1.5) is positive in the sense that no partial population is negative at any time if its initial conditions are nonnegative and the vaccinated population exceeds a certain minimum measurable threshold in the event that the recovered population is zero as follows: $V(t) \geq \max \left(\left(\gamma / \gamma_{1}\right)\left(I(t-\omega) e^{-b \omega}-I(t)\right), 0\right)$ if $R(t)=0$. The susceptible, vaccinated, exposed, and infected populations are nonnegative for all time irrespective of the above constraint. If, in addition, Theorem 4.2(i) holds, then all the partial populations of the SVEIRS model are uniformly bounded for all time.

Proof. First note that all the partial populations are defined by continuous-time differentiable functions from (1.1)-(1.5). Then, if any partial population is negative, it is zero at some previous time instant. Assume that $S(\sigma) \geq 0$ for $\sigma<t$ and $S(t)=0$ at some time instant $t$. Then, from (1.1)

$$
\dot{S}(t)=b+\gamma I(t-\omega) e^{-b \omega}+v\left(1-V_{c}\right) N(t) \geq 0 ; \quad \forall V_{c} \in[0,1] .
$$

Thus, $S\left(t^{+}\right) \geq 0$. As a result, $S(t)$ cannot reach negative values at any time instant. Assume that $V(\sigma) \geq 0$ for $\sigma<t$ and $V(t)=0$ at some time instant $t$. Then, $\dot{V}(t)=v V_{c} N(t) \geq 0$ from (1.2) so that $V\left(t^{+}\right) \geq 0$. As a result, $V(t)$ cannot reach negative values at any time. $E(t) \geq 0$ for any time instant $t$ from (1.3). Assume that $I(\sigma) \geq 0$ for $\sigma<t$ and $I(t)=0$ at some time instant $t$. Then, $\dot{I}(t) \geq 0$ from (1.4). As a result, $I(t)$ cannot reach negative values at any time. Finally, assume that $R(\sigma) \geq 0$ for $\sigma<t$ and $R(t)=0$ at some time instant $t$. Thus, $\dot{R}(t)=$ $\gamma_{1} V(t)+\gamma\left(I(t)-I(t-\omega) e^{-b \omega}\right) \geq 0$ from (1.5) if $V(t) \geq \max \left(\left(\gamma / \gamma_{1}\right)\left(I(t-\omega) e^{-b \omega}-I(t)\right), 0\right)$. Thus, 
if $V(t) \geq \max \left(\left(\gamma / \gamma_{1}\right)\left(I(t-\omega) e^{-b \omega}-I(t)\right), 0\right)$ when $R(t)=0$, then all the partial populations are uniformly bounded, since they are nonnegative and the total population $N(t)$ is uniformly bounded from Theorem 4.2(i).

It is discussed in the next section that if the two above theorems related to positivity and boundedness hold, then the solution trajectories converge to either the disease-free equilibrium point or to the endemic equilibrium point.

\section{Solution Trajectory of the SVEIRS Model}

The solution trajectories of the SVEIRS differential model (1.1)-(1.5) are given below.

Equation (1.1) yields

$$
\begin{aligned}
S(t)= & e^{-\int_{0}^{t}(b+\beta(I(\xi) /(1+\eta S(\xi)))) d \xi} S(0) \\
& +\int_{0}^{t} e^{-\int_{\xi}^{t}(b+\beta(I(\sigma) /(1+\eta S(\sigma)))) d \sigma}\left(\gamma I(\xi-\omega) e^{-b \omega}+v\left(1-V_{c}\right) N(\xi)+b\right) d \xi .
\end{aligned}
$$

Equation (1.2) yields

$$
V(t)=e^{-\int_{0}^{t}\left(\gamma_{1}+b+(\delta \beta I(\xi) /(1+\eta V(\xi)))\right) d \xi} V(0)+\mathcal{v} V_{c} \int_{0}^{t} e^{-\int_{\xi}^{t}\left(\gamma_{1}+b+(\delta \beta I(\sigma) /(1+\eta V(\sigma)))\right) d \sigma} N(\xi) d \xi
$$

Equation (1.3) is already in integral form. Equation (1.4) yields

$$
\begin{aligned}
I(t) & =e^{-(\gamma+b+\alpha) t}\left[I(0)+\beta e^{-b \tau} \int_{0}^{t} e^{(\gamma+b+\alpha) \xi}\left(\frac{S(\xi-\tau)}{1+\eta S(\xi-\tau)}+\frac{\delta V(\xi-\tau)}{1+\eta V(\xi-\tau)}\right) I(\xi-\tau) d \xi\right] \\
& \leq e^{-(\gamma+b+\alpha) t}\left[I(0)+\frac{1+\delta}{\eta} \beta e^{-b \tau} \int_{0}^{t} e^{(\gamma+b+\alpha) \xi} I(\xi-\tau) d \xi\right] .
\end{aligned}
$$

Equation (1.5) yields

$$
R(t)=e^{-b t}\left[R(0)+\int_{0}^{t} e^{b \xi}\left(\gamma_{1} V(\xi)+\gamma\left(I(\xi)-I(\xi-\omega) e^{-b \omega}\right)\right) d \xi\right] .
$$

The asymptotic values of the partial populations can be calculated from (5.1)-(5.4) as time tends to infinity as follows provided that the involved right-hand-side integrals exist:

$$
\begin{gathered}
S(\infty)=\int_{0}^{\infty} e^{-\int_{\xi}^{\infty}(b+\beta(I(\sigma) /(1+\eta S(\sigma)))) d \sigma}\left(\gamma I(\xi-\omega) e^{-b \omega}+v\left(1-V_{c}\right) N(\xi)+b\right) d \xi \\
V(\infty)=v V_{c} \int_{0}^{\infty} e^{-\int_{\xi}^{\infty}\left(\gamma_{1}+b+(\delta \beta I(\sigma) /(1+\eta V(\sigma)))\right) d \sigma} N(\xi) d \xi
\end{gathered}
$$




$$
\begin{gathered}
E(\infty)=\frac{\beta}{b}\left(1-e^{-b \omega}\right)\left(\frac{S(\infty)}{1+\eta S(\infty)}+\frac{\delta V(\infty)}{1+\eta V(\infty)}\right) I(\infty) \\
I(\infty)=\beta e^{-b \tau} e^{-(\gamma+b+\alpha) t} \int_{0}^{\infty} e^{(\gamma+b+\alpha) \xi}\left(\frac{S(\xi-\tau)}{1+\eta S(\xi-\tau)}+\frac{\delta V(\xi-\tau)}{1+\eta V(\xi-\tau)}\right) I(\xi-\tau) d \xi, \\
R(\infty)=\lim _{t \rightarrow \infty}\left(\int_{0}^{t} e^{-b(t-\xi)}\left(\gamma_{1} V(\xi)+\gamma\left(I(\xi)-I(\xi-\tau) e^{-b \omega}\right)\right) d \xi\right) .
\end{gathered}
$$

Remark 5.1. If any of the above right-hand-side integrals with upper-limit $+\infty$ does not exist, then the corresponding limit of the involved partial population as time tends to infinity does not exist and, then, the limit value has to be replaced by the existing limit superior as time tends to infinity. Note that if Theorems 4.2 and 4.3 hold then all the limit values at infinity of the partial populations exist since the total population is uniformly bounded and all the partial populations are nonnegative for all time. A conclusion of this feature is that under positivity and boundedness of the solutions, all the partial populations of the impulsivefree SVEIRS model (1.1)-(1.5) have finite limits as time tends to infinity. As a result, all the trajectory solutions converge asymptotically either to the disease-free equilibrium point or to the endemic equilibrium point.

The considerations in Remark 5.1 are formally expressed as the subsequent important result by taking also into account the uniqueness of the infected population at any endemic equilibrium points, the uniqueness of the vaccinated population at such points (which follows from (3.12) and which implies the uniqueness of the total population at such an equilibrium endemic points (see Remark 5.1).

Theorem 5.2. The following two properties hold.

(i) The endemic equilibrium point is unique.

(ii) Assume that Theorems 4.2 and 4.3 hold. Then, any solution trajectory of the SVEIRS impulsive-free vaccination model (1.1)-(1.5) generated for finite initial conditions converges asymptotically either to the disease-free equilibrium point or to the endemic equilibrium point as time tends to infinity.

Now, assume that the solution trajectory converges to some locally asymptotically stable equilibrium point $\left(S^{*}, V^{*}, E^{*}, I^{*}, R^{*}\right)^{T}$. The above equations ensure the existence of integrands $f_{S} \in L_{1}\left(\mathbf{R}_{0+}\right), f_{V} \in L_{1}\left(\mathbf{R}_{0+}\right), f_{I} \in L_{1}\left(\mathbf{R}_{0+}\right)$, being nonnegative, and $f_{R}(t)=O\left(e^{b t}\right)$ such that $f_{R}(t) e^{-b t} \leq K_{R}(t)$ for some $K_{R} \in L_{1}\left(\mathbf{R}_{0+}\right)$, for any initial conditions in some small neighbourhood of the equilibrium, such that

$$
\begin{gathered}
S(\infty)=S^{*}=\int_{0}^{\infty} f_{S}(t) d t ; \quad V(\infty)=V^{*}=\int_{0}^{\infty} f_{V}(t) d t, \quad I(\infty)=I^{*}=\int_{0}^{\infty} f_{I}(t) d, \\
R(\infty)=R^{*}=\lim _{t \rightarrow \infty}\left(\int_{0}^{t} e^{-b t} f_{R}(\xi) d \xi\right),
\end{gathered}
$$


which leads to

$$
\begin{gathered}
\gamma_{1} V(t)+\gamma\left(I(t)-I(t-\tau) e^{-b \omega}\right) \\
\leq f_{R}(t) e^{-b t} \leq K_{R}(t) \Longrightarrow-\gamma I(t-\omega) e^{-b \omega} \leq K_{R}(t+\tau-\omega)-\gamma_{1} V(t+\tau-\omega) \\
\quad-\gamma I(t+\tau-\omega), \\
N(t)=\frac{1}{v V_{c}}\left(e^{\int_{t}^{\infty}\left(\gamma_{1}+b+(\delta \beta I(\sigma) /(1+\eta V(\sigma)))\right) d \sigma}\right) f_{V}(t) \\
=\frac{1}{v\left(1-V_{c}\right)}\left[\left(e^{\int_{t}^{\infty}(b+(\beta I(\sigma) /(1+\eta S(\sigma)))) d \sigma}\right) f_{S}(t)-\gamma I(t-\omega) e^{-b \omega}-b\right] \\
\leq \frac{1}{v\left(1-V_{c}\right)}\left[\left(e^{\int_{t}^{\infty}(b+(\beta I(\sigma) /(1+\eta S(\sigma)))) d \sigma}\right) f_{S}(t)+K_{R}(t+\tau-\omega)\right. \\
\left.\quad-\gamma_{1} V(t+\tau-\omega)-\gamma I(t+\tau-\omega)-b\right] .
\end{gathered}
$$

Any equilibrium point also satisfies the following constraints from (5.1)-(5.4), and (1.3) after performing the replacements $0 \rightarrow k T$ (for the initial time instant), $t \rightarrow(k+1) T$ for the final time instant, for any given $T \in \mathbf{R}_{0+}$, and taking limits as $k\left(\in \mathbf{Z}_{0+}\right) \rightarrow \infty S(k T)=S[(k+1) T]=$ $S^{*}, V(k T)=V[(k+1) T]=V^{*}, E(k T)=E[(k+1) T]=E^{*}, I(k T)=I[(k+1) T]=I^{*}, R(k T)=$ $R[(k+1) T]=R^{*}$, and $N(k T)=N[(k+1) T]=N^{*}$. This leads to the following implicit relations being independent of $T$ and being applicable for any (disease-free or endemic) equilibrium point:

$$
\begin{aligned}
S^{*}= & \left(\gamma I^{*} e^{-b \omega}+v\left(1-V_{c}\right) N^{*}+b\right)\left(1-e^{-\left(b+\beta\left(I^{*} /\left(1+\eta S^{*}\right)\right)\right) T}\right)^{-1} \\
& \times\left(\lim _{k\left(\in \mathbf{Z}_{0+}\right) \rightarrow \infty} \int_{k T}^{(k+1) T} e^{-\int_{\xi}^{(k+1) T}\left(b+\beta\left(I^{*} /\left(1+\eta S^{*}\right)\right)\right) d \sigma} d \xi\right) \\
= & \frac{\gamma I^{*} e^{-b \omega}+\mathcal{v}\left(1-V_{c}\right) N^{*}+b}{b+\beta\left(I^{*} /\left(1+\eta S^{*}\right)\right)} .
\end{aligned}
$$

Equation (1.2) yields

$$
\begin{aligned}
& V^{*}=v V_{c} N^{*}\left(1-e^{-\left(\gamma_{1}+b+\left(\delta \beta I^{*} /\left(1+\eta V^{*}\right)\right)\right) T}\right)^{-1} \lim _{k\left(\in \mathbf{Z}_{0+}\right) \rightarrow \infty}\left(\int_{k T}^{(k+1) T} e^{-\left(\gamma_{1}+b+\left(\delta \beta I^{*} /\left(1+\eta V^{*}\right)\right)\right)((k+1) T-\xi)} d \xi\right) \\
&= \frac{v V_{c} N^{*}}{\gamma_{1}+b+\left(\delta \beta I^{*} /\left(1+\eta V^{*}\right)\right)}, \\
& E^{*}=\frac{\beta}{b}\left(1-e^{-b \omega}\right)\left(\frac{S^{*}}{1+\eta S^{*}}+\frac{\delta V^{*}}{1+\eta V^{*}}\right) I^{*}
\end{aligned}
$$


Equation (1.4) yields

$$
\begin{aligned}
I^{*} & =\beta e^{-b \tau}\left(1-e^{-(\gamma+b+\alpha) T}\right)^{-1}\left(\frac{S^{*} I^{*}}{1+\eta S^{*}}+\frac{\delta V^{*} I^{*}}{1+\eta V^{*}}\right)\left(\lim _{k\left(\in \mathbf{Z}_{0_{+}} \rightarrow \infty\right.} \int_{k T}^{(k+1) T} e^{-(\gamma+b+\alpha)((k+1) T-\xi)} d \xi\right) \\
& =\frac{\beta e^{-b \tau} I^{*}}{\gamma+b+\alpha}\left(\frac{S^{*}}{1+\eta S^{*}}+\frac{\delta V^{*}}{1+\eta V^{*}}\right),
\end{aligned}
$$

which is identical to (2.5), that is, either $I^{*}=0$ or $1=\left(\beta e^{-b \tau} I^{*} /(\gamma+b+\alpha)\right)\left(S^{*} /\left(1+\eta S^{*}\right)+\right.$ $\left.\delta V^{*} /\left(1+\eta V^{*}\right)\right)$. Equation (1.5) yields

$$
\begin{aligned}
R^{*} & =\left(1-e^{-b T}\right)^{-1}\left(\gamma_{1} V^{*}+\gamma\left(1-e^{-b \omega}\right) I^{*}\right)\left(\lim _{k\left(\in \mathbf{Z}_{0+}\right) \rightarrow \infty} \int_{k T}^{(k+1) T} e^{-b((k+1) T-\xi)} d \xi\right) \\
& =\frac{1}{b}\left(\gamma_{1} V^{*}+\gamma\left(1-e^{-b \omega}\right) I^{*}\right),
\end{aligned}
$$

which is identical to (2.6).

Remark 5.3. Some fast observations useful for the model interpretation follow by simple inspection of (5.1)-(5.4) and (5.10).

(1) If $b>0$ or $V_{c}<1$, then $S(t) \rightarrow 0$ as $t \rightarrow \infty$ is impossible.

(2) $V(t) \rightarrow 0$ as $t \rightarrow \infty$ occurs if $V_{c}$ is eventually a function of time, rather than a real constant, subject to $V_{c}(t) \leq K N^{-1}(t) e^{-\int_{0}^{t}\left(\gamma_{1}+b+(\delta \beta I(\sigma) /(1+\eta V(\sigma))) d \sigma\right)}$ for some $K \in \mathbf{R}_{+}$, for almost all $t \in \mathbf{R}_{0+}$ except possibly at a set of zero measure in the case that the total population does not extinguish.

(3) $I(t)$ can converge to zero exponentially with time, for instance, to the disease-free equilibrium point, while being a function of exponential order $-(\gamma+b+\alpha)$ and, in such a case, $E(t)$ also converges to zero exponentially while being of exponential order $-(\gamma+\alpha)$ and satisfying $E(t) \leq K^{\prime} \omega e^{(\gamma+b+\alpha) \omega}((1+\delta) / \eta) e^{-(\gamma+\alpha) t}$ from (1.3).

(4) $R(t) \rightarrow 0$ as $t \rightarrow \infty$ requires from (5.4) the two above corresponding conditions for the infective and vaccinated populations to converge to zero. In such a case, the convergence of the recovered population to zero is also at an exponential rate. An alternative condition for a convergence to zero of the recovered population, perhaps at a rate slower than exponential, is the convergence to zero of the function

$$
V(t)+\left(\frac{\gamma}{\gamma_{1}}\right)\left(I(t)-I(t-\omega) e^{-b \omega}\right)
$$

(see (5.4) with alternating sign on any two consecutive appropriate time intervals of finite lengths). 


\subsection{Incorporation of Impulsive Vaccination to the SVEIRS Model}

Impulsive vaccination involving culling-type or removal actions on the susceptible population has being investigated recently in $[18,19]$. It has also being investigated in [20] in the context of a very general SEIR model. Assume that the differential system (1.1)-(1.5) is used for modelling in open real $\left(t_{k}, t_{k+1}\right)$ intervals for some given real sequence of time intervals $\left\{T_{k}\right\}_{k \in \mathbf{Z}_{0+}}$ with $T_{k} \geq T_{\min }>0$, for all $k \in \mathbf{Z}_{0+}$ and an impulsive vaccination is used at time instants $t_{k}=t_{k-1}+T_{k-1}=\sum_{j=0}^{k-1} T_{j}, t_{0} \geq 0$ in the sequence $\left\{t_{k}\right\}_{k \in \mathbf{Z}_{0+}}$ which leads to a susceptible culling (or partial removal of susceptible from the habitat) and corresponding vaccinated increase as follows for some given real sequence $\left\{\theta_{k}\right\}_{k \in \mathbf{Z}_{0+}}$, where $\theta_{k} \in[0,1]$ :

$$
\begin{gathered}
S\left(t_{k}^{+}\right)=\left(1-\theta_{k}\right) S\left(t_{k}\right) ; \quad V\left(t_{k}^{+}\right)=V\left(t_{k}\right)+\theta_{k} S\left(t_{k}\right), \\
E\left(t_{k}^{+}\right)=E\left(t_{k}\right) ; \quad I\left(t_{k}^{+}\right)=I\left(t_{k}\right) ; \quad R\left(t_{k}^{+}\right)=R\left(t_{k}\right),
\end{gathered}
$$

so that $S\left(t_{k}^{+}\right)+V\left(t_{k}^{+}\right)=S\left(t_{k}\right)+V\left(t_{k}\right)$. The following simple result follows trivially.

Theorem 5.4. Let $\left\{t_{k}\right\}_{k \in \mathbf{Z}_{0^{+}}}$and $\left\{\theta_{k}\right\}_{k \in \mathbf{Z}_{0+}}$ be arbitrary except that the second one has all its elements in $[\varepsilon, 1]$ for some $\varepsilon(<1) \in \mathbf{R}_{+}$. Then, there is no nonzero equilibrium point of the impulsive SVEIRS model (1.1)-(1.5), (5.14). If $\theta_{k} \rightarrow 0$ as $k \rightarrow \infty$, then the equilibrium points of the impulsive model are the same as those of the SVEIRS model (1.1)-(1.5).

Equations (5.1), (5.2) yield to the following recursive expressions:

$$
\begin{aligned}
S\left(t_{k}+\rho\right)= & e^{-\int_{0}^{\rho}\left(b+\beta\left(I\left(t_{k}+\xi\right) /\left(1+\eta S\left(t_{k}+\xi\right)\right)\right) d \xi\right.} S\left(t_{k}^{+}\right) \\
& +\int_{0}^{\rho} e^{-\int_{\xi}^{\rho}\left(b+\beta\left(I\left(t_{k}+\sigma\right) /\left(1+\eta S\left(t_{k}+\sigma\right)\right)\right)\right) d \sigma} \\
& \quad \times\left(\gamma I\left(t_{k}+\xi-\omega\right) e^{-b \omega}+v\left(1-V_{c}\right) N\left(t_{k}+\xi\right)+b\right) d \xi ; \quad \forall \rho \in\left[0, T_{k}\right) \\
S\left(t_{k+1}^{+}\right)= & \left(1-\theta_{k+1}\right) S\left(t_{k+1}\right)=\left(1-\theta_{k+1}\right) e^{-\int_{0}^{T_{k}}\left(b+\beta\left(I\left(t_{k}+\xi\right) /\left(1+\eta S\left(t_{k}+\xi\right)\right)\right)\right) d \xi} S\left(t_{k}^{+}\right) \\
& +\left(1-\theta_{k+1}\right) \int_{0}^{T_{k}} e^{-\int_{\xi}^{T_{k}}\left(b+\beta\left(I\left(t_{k}+\sigma\right) /\left(1+\eta S\left(t_{k}+\sigma\right)\right)\right)\right) d \sigma} \\
& \times\left(\gamma I\left(t_{k}+\xi-\omega\right) e^{-b \omega}+v\left(1-V_{c}\right) N\left(t_{k}+\xi\right)+b\right) d \xi \\
V\left(t_{k}+\rho\right)= & e^{-\int_{0}^{\rho}\left(\gamma_{1}+b+\left(\delta \beta I\left(t_{k}+\xi\right) /\left(1+\eta V\left(t_{k}+\xi\right)\right)\right)\right) d \xi} V\left(t_{k}^{+}\right)+v V_{c} \int_{0}^{\rho} e^{-\int_{\xi}^{\rho}\left(\gamma_{1}+b+\left(\delta \beta I\left(t_{k}+\sigma\right) /\left(1+\eta V\left(t_{k}+\sigma\right)\right)\right)\right) d \sigma}
\end{aligned}
$$




$$
\begin{aligned}
V\left(t_{k+1}^{+}\right)= & \theta_{k+1} S\left(t_{k+1}\right)+V\left(t_{k+1}\right)=\theta_{k+1} e^{-\int_{0}^{T_{k}}\left(b+\beta\left(I\left(t_{k}+\xi\right) /\left(1+\eta S\left(t_{k}+\xi\right)\right)\right)\right) d \xi} S\left(t_{k}^{+}\right)+\theta_{k+1} \\
& \times \int_{0}^{T_{k}} e^{-\int_{\xi}^{T_{k}}\left(b+\beta\left(I\left(t_{k}+\sigma\right) /\left(1+\eta S\left(t_{k}+\sigma\right)\right)\right)\right) d \sigma}\left(\gamma I\left(t_{k}+\xi-\omega\right) e^{-b \omega}+v\left(1-V_{c}\right) N\left(t_{k}+\xi\right)+b\right) d \xi \\
+ & e^{-\int_{0}^{T_{k}}\left(\gamma_{1}+b+\left(\delta \beta I\left(t_{k}+\xi\right) /\left(1+\eta V\left(t_{k}+\xi\right)\right)\right)\right) d \xi} V\left(t_{k}^{+}\right)+v V_{c} \int_{0}^{T_{k}} e^{-\int_{\xi}^{T_{k}}\left(\gamma_{1}+b+\left(\delta \beta I\left(t_{k}+\sigma\right) /\left(1+\eta V\left(t_{k}+\sigma\right)\right)\right)\right) d \sigma} \\
& \times N\left(t_{k}+\xi\right) d \xi .
\end{aligned}
$$

The solutions in-between two consecutive impulsive vaccinations are obtained by slightly modifying (5.15)-(5.18) by replacing $\theta_{k}$ by zero and $t_{k+1}$ by $t_{k}+\rho<t_{k+1}$ for $\rho \in\left[0, T_{k}\right.$ ). The following result about the oscillatory behaviour of the vaccinated population holds.

Theorem 5.5. Assume that $V_{c}: \mathbf{R}_{0+} \rightarrow[0,1]$ is a piecewise continuous function rather than a constant one with eventual bounded step discontinuities at the sequence of impulsive time instants. Define real sequences at impulsive time instants with general terms

$$
\begin{gathered}
V_{c 1}\left(t_{k}\right):=\frac{V\left(t_{k}^{+}\right)}{v \int_{0}^{\infty} e^{-\int_{\xi}^{\infty}\left(\gamma_{1}+b+\beta\left(I\left(t_{k}+\sigma\right) /\left(1+\eta S\left(t_{k}+\sigma\right)\right)\right)\right) d \sigma} N\left(t_{k}+\xi\right) d \xi}, \\
V_{c 2}\left(t_{k}\right):=\frac{V\left(t_{k}^{+}\right)-\int_{0}^{\infty} e^{-\int_{\xi}^{\infty}\left(b+\beta\left(I\left(t_{k}+\sigma\right) /\left(1+\eta S\left(t_{k}+\sigma\right)\right)\right)\right) d \sigma}\left(\gamma I\left(t_{k}+\xi-\omega\right) e^{-b \omega}+v N\left(t_{k}+\xi\right)+b\right) d \xi}{v \int_{0}^{\infty}\left(e^{-\int_{\xi}^{\infty}\left(\gamma_{1}+b+\left(\delta \beta I\left(t_{k}+\sigma\right) /\left(1+\eta V\left(t_{k}+\sigma\right)\right)\right)\right) d \sigma}-e^{-\int_{\xi}^{\infty}\left(b+\beta\left(I\left(t_{k}+\sigma\right) /\left(1+\eta S\left(t_{k}+\sigma\right)\right)\right)\right) d \sigma}\right) N\left(t_{k}+\xi\right) d \xi} .
\end{gathered}
$$

Then, the vaccinated population is an oscillating function if there is a culling sequence $\left\{t_{k}\right\}_{k \in \mathbf{Z}_{0+}}$ of time instants for some given real sequence $\left\{\theta_{k}\right\}_{k \in \mathbf{Z}_{0+}}$ with $\theta_{k} \in[0,1]$, of impulsive gains if any two consecutive impulsive time instants satisfy some of the two conditions below.

Condition 1. $V_{c 1}\left(t_{k}\right) \leq 1$ and $0 \leq V_{c 2}\left(t_{k+1}\right) \leq 1$ with a regular piecewise continuous vaccination $V_{c}\left(t_{k}\right) \in\left[V_{c 1}\left(t_{k}\right), 1\right]$ and $V_{c}\left(t_{k+1}\right) \in\left[0, V_{c 2}\left(t_{k+1}\right)\right]$.

Condition 2. $0 \leq V_{c 1}\left(t_{k+1}\right) \leq 1$ and $V_{c 2}\left(t_{k}\right) \leq 1$ with a regular piecewise continuous vaccination $V_{c}\left(t_{k}\right) \in\left[0, V_{c 1}\left(t_{k}\right)\right]$ and $V_{c}\left(t_{k+1}\right) \in\left[V_{c 2}\left(t_{k+1}\right), 1\right]$.

Proof. One gets directly by using lower and upper-bounds in (5.18) via $\theta_{k+1}=0$ and $\theta_{k+1}=1$ for $V\left(t_{k+1}^{+}\right)-V\left(t_{k}^{+}\right)$

$$
\begin{aligned}
v V_{c}\left(t_{k}\right) & \int_{0}^{T_{k}} e^{-\int_{\xi}^{T_{k}}\left(\gamma_{1}+b+\left(\delta \beta I\left(t_{k}+\sigma\right) /\left(1+\eta V\left(t_{k}+\sigma\right)\right)\right)\right) d \sigma} N\left(t_{k}+\xi\right) d \xi \\
& -\left(1-e^{-\int_{0}^{T_{k}}\left(\gamma_{1}+b+\left(\delta \beta I\left(t_{k}+\xi\right) /\left(1+\eta V\left(t_{k}+\xi\right)\right)\right)\right) d \xi}\right) V\left(t_{k}^{+}\right) \\
\leq & V\left(t_{k+1}^{+}\right)-V\left(t_{k}^{+}\right) \leq e^{-\int_{0}^{T_{k}}\left(b+\beta\left(I\left(t_{k}+\xi\right) /\left(1+\eta S\left(t_{k}+\xi\right)\right)\right)\right) d \xi} S\left(t_{k}^{+}\right) \\
& +\int_{0}^{T_{k}} e^{-\int_{\xi}^{T_{k}}\left(b+\beta\left(I\left(t_{k}+\sigma\right) /\left(1+\eta S\left(t_{k}+\sigma\right)\right)\right)\right) d \sigma}\left(\gamma I\left(t_{k}+\xi-\omega\right) e^{-b \omega}+v\left(1-V_{c}\right) N\left(t_{k}+\xi\right)+b\right) d \xi \\
& +v V_{c}\left(t_{k}\right) \int_{0}^{T_{k}} e^{-\int_{\xi}^{T_{k}}\left(\gamma_{1}+b+\left(\delta \beta I\left(t_{k}+\sigma\right) /\left(1+\eta V\left(t_{k}+\sigma\right)\right)\right)\right) d \sigma} N\left(t_{k}+\xi\right) d \xi \\
& -\left(1-e^{-\int_{0}^{T_{k}}\left(\gamma_{1}+b+\left(\delta \beta I\left(t_{k}+\xi\right) /\left(1+\eta V\left(t_{k}+\xi\right)\right)\right)\right) d \xi}\right) V\left(t_{k}^{+}\right)
\end{aligned}
$$


so that as $T_{k} \rightarrow \infty$ and also for some sufficiently large $T_{k}=t_{k+1}-t_{k}$,

$$
\begin{aligned}
& \liminf _{T_{k} \rightarrow \infty}\left(V\left(t_{k+1}^{+}\right)-V\left(t_{k}^{+}\right)\right) \\
& \quad \geq v V_{c}\left(t_{k}\right) \int_{0}^{\infty} e^{-\int_{\xi}^{\infty}\left(\gamma_{1}+b+\beta\left(I\left(t_{k}+\sigma\right) /\left(1+\eta S\left(t_{k}+\sigma\right)\right)\right)\right) d \sigma} N\left(t_{k}+\xi\right) d \xi-V\left(t_{k}^{+}\right)>0,
\end{aligned}
$$

if $V_{c}\left(t_{k}\right) \in\left(V_{c 1}\left(t_{k}\right), \infty\right)$, and

$$
\begin{aligned}
\limsup _{T_{k} \rightarrow \infty} & \left(V\left(t_{k+1}^{+}\right)-V\left(t_{k}^{+}\right)\right) \\
\leq & e^{-\int_{0}^{\infty}\left(b+\left(\beta I\left(t_{k}+\sigma\right) /\left(1+\eta S\left(t_{k}+\sigma\right)\right)\right) d \sigma\right)} \\
& +\int_{0}^{\infty} e^{-\int_{\xi}^{\infty}\left(b+\beta\left(I\left(t_{k}+\sigma\right) /\left(1+\eta S\left(t_{k}+\sigma\right)\right)\right)\right) d \sigma}\left(\gamma I\left(t_{k}+\xi-\omega\right) e^{-b \omega}+v N\left(t_{k}+\xi\right)+b\right) d \xi \\
+ & v V_{c}\left(t_{k}\right) \int_{0}^{\infty}\left(e^{-\int_{\xi}^{\infty}\left(\gamma_{1}+b+\left(\delta \beta I\left(t_{k}+\sigma\right) /\left(1+\eta V\left(t_{k}+\sigma\right)\right)\right)\right) d \sigma}-e^{-\int_{\xi}^{\infty}\left(b+\beta\left(I\left(t_{k}+\sigma\right) /\left(1+\eta S\left(t_{k}+\sigma\right)\right)\right)\right) d \sigma}\right) \\
& \times N\left(t_{k}+\xi\right) d \xi-V\left(t_{k}^{+}\right)<0
\end{aligned}
$$

if $V_{c}\left(t_{k}\right) \in\left(-\infty, V_{c 2}\left(t_{k}\right)\right)$. Thus, $V_{c 1}\left(t_{k}\right) \leq 1$ and $0 \leq V_{c 2}\left(t_{k+1}\right) \leq 1$ imply that $V\left(t_{k+1}^{+}\right)>V\left(t_{k}^{+}\right)$ and $V\left(t_{k+2}^{+}\right)<V\left(t_{k+1}^{+}\right)$, and $0 \leq V_{c 1}\left(t_{k}\right) \leq 1$ and $V_{c 2}\left(t_{k+1}\right) \leq 1$ imply that $V\left(t_{k+1}^{+}\right)<V\left(t_{k}^{+}\right)$and $V\left(t_{k+2}^{+}\right)>V\left(t_{k+1}^{+}\right)$for some $\theta_{k}, \theta_{k+1} \in[0,1]$ and some sufficiently large intervals in-between consecutive impulses $T_{k}$ and $T_{k+1}$ via the use of an admissible regular piecewise continuous vaccination $V_{c}\left(t_{k}\right) \in\left[V_{c 1}\left(t_{k}\right), 1\right]$ and $V_{c}\left(t_{k+1}\right) \in\left[0, V_{c 2}\left(t_{k+1}\right)\right]$.

It turns out that Theorem 5.5 might be generalized by grouping a set of consecutive impulsive time instants such that $V\left(t_{k+i+1}^{+}\right)>V\left(t_{k}^{+}\right)$and $V\left(t_{k+i+j+1}^{+}\right)<V\left(t_{k+i+1}^{+}\right)$for some positive integers $i=i(k), j=j(i, k)$.

A further property now described is that of the impulsive-free infection permanence in the sense that for sufficiently large initial conditions of the infected populations the infected population exceeds a, initial conditions dependent, positive lower-bound for all time if no impulsive vaccination is injected under any regular vaccination.

Theorem 5.6. Assume that the SVEIRS model is positive (in the sense that no partial population reaches a negative value for any time under nonnegative initial conditions) and, furthermore, that the susceptible and vaccinated populations remain positive for all time. Assume also that the disease transmission constant is subject to the subsequent constraint

$$
\frac{g_{\beta 2} e^{b \tau}(\gamma+\beta+\alpha)\left[\eta+\max \left(u_{S}^{-1}, u_{V}^{-1}\right)\right]}{1+\delta} \geq \beta \geq \frac{g_{\beta 1} e^{b \tau}(\gamma+\beta+\alpha)\left[\eta+\max \left(u_{S}^{-1}, u_{V}^{-1}\right)\right]}{1+\delta},
$$

for some positive real constants dependent on the delay $\tau$

$$
g_{\beta 1}=g_{\beta 1}(\tau) \geq 1, \quad g_{\beta 2}=g_{\beta 2}(\tau) \geq \max \left(g_{\beta 1}, \frac{\tilde{g}-e^{-(\gamma+b+\alpha) \tau}}{1-e^{-(\gamma+b+\alpha) \tau}}\right), \quad \tilde{g}=\tilde{g}(\tau) \geq 1 .
$$

Then, the infection is permanent for all time if it strictly exceeds zero along the initialization interval. 
Proof. Note from the solution trajectories of the susceptible and vaccinated populations (5.1) and (5.2) that for some $\inf _{t \in \mathbf{R}_{0+}} S(t) \geq u_{S}>0$ and $\inf _{t \in \mathbf{R}_{0+}} V(t) \geq u_{V}>0$ for some real constants $u_{S}>0$ and $u_{V}>0$, if $b>0$ and $V_{c} \in(0,1]$. Then,

$$
\begin{aligned}
\frac{S(t)}{1+\eta S(t)}+\frac{\delta V(t)}{1+\eta V(t)} & =\frac{1}{S^{-1}(t)+\eta}+\frac{\delta}{V^{-1}(t)+\eta} \geq \frac{1}{u_{S}^{-1}+\eta}+\frac{\delta}{u_{V}^{-1}+\eta} \\
& \geq \frac{1+\delta}{\eta+\max \left(u_{S}^{-1}, u_{V}^{-1}\right)} ; \quad \forall t \in[-\tau, \infty),
\end{aligned}
$$

so that, from (5.3a), since the SVEIR model is positive

$$
\begin{aligned}
I(t+\sigma) \geq & e^{-(\gamma+b+\alpha)(\sigma+\tau)} I(t-\tau)+\frac{\beta e^{-b \tau}(1+\delta)}{(\gamma+b+\alpha)\left[\eta+\max \left(u_{S}^{-1}, u_{V}^{-1}\right)\right]} \\
& \times\left(1-e^{-(\gamma+b+\alpha)(\tau+\sigma)}\right)\left(\inf _{t-2 \tau \leq \xi \leq t-\tau+\sigma} I(\xi)\right) \\
\geq & \left(e^{-(\gamma+b+\alpha)(\tau+\sigma)}+\frac{\beta e^{-b \tau}(1+\delta)}{(\gamma+b+\alpha)\left[\eta+\max \left(u_{S}^{-1}, u_{V}^{-1}\right)\right]}\left(1-e^{-(\gamma+b+\alpha)(\tau+\sigma)}\right)\right) \\
& \times\left(\inf _{t-2 \tau \leq \xi \leq t-\tau+\sigma} I(\xi)\right) \\
\geq & \left(\frac{\beta e^{-b \tau}(1+\delta)}{(\gamma+b+\alpha)\left[\eta+\min \left(u_{S}^{-1}, u_{V}^{-1}\right)\right]}-\left(\frac{\beta e^{-b \tau}(1+\delta)}{(\gamma+b+\alpha)\left[\eta+\max \left(u_{S}^{-1}, u_{V}^{-1}\right)\right]}-1\right)\right. \\
& \left.\quad \times e^{-(\gamma+b+\alpha) \tau}\right)\left(\inf _{t-2 \tau \leq \xi \leq t-\tau+\sigma} I(\xi)\right) \\
\geq & \left(g_{\beta 1}-\left(g_{\beta 2}-1\right) e^{-(\gamma+b+\alpha) \tau}\right)\left(\inf _{t-2 \tau \leq \xi \leq t-\tau+\sigma} I(\xi)\right) \geq \widetilde{g}\left(\inf _{t-2 \tau \leq \xi \leq t-\tau+\sigma} I(\xi)\right) \\
\geq & \tilde{g}_{t-2 \tau \leq \xi \leq t-\tau+\sigma} I(\xi)=\operatorname{ginf}_{\tau \leq \xi \leq 0} I(\xi) \geq \min \left(g_{\beta 1}, \tilde{g}\right) \inf _{\tau \leq \xi \leq 0} I(\xi) ; \quad \forall \sigma \in \mathbf{R}_{0+}, \forall t \in \mathbf{R}_{0+} .
\end{aligned}
$$

The two last inequalities following from the fact that the former one stands, for all $t \in$ $\mathbf{R}_{0+}$ (an "ad-hoc" complete induction reasoning will lead to an identical conclusion), and, furthermore,

$$
\liminf _{t \rightarrow \infty} I(t+\sigma) \geq g_{\beta 1} \inf _{\tau \leq \xi \leq 0} I(\xi) \geq \min \left(g_{\beta 1}, \widetilde{g}\right) \inf _{\tau \leq \xi \leq 0} I(\xi)
$$

Thus, the infection is permanent for all time if it is permanent for the initialization time interval $[-\tau, 0]$.

It is also obvious the following simplification of Theorem 5.6. 
Theorem 5.7. Theorem 5.6 still holds under (5.24) and the "ad-hoc" modified inequality (5.23) if either the susceptible or the vaccinated (but not both) population reaches zero in finite time or tends to zero asymptotically.

Proof. It is similar to that of Theorem 5.6 by zeroing either $u_{S}$ and $u_{V}$ and removing the inverses from the corresponding conditions and proofs.

Close to the above results is the asymptotic permanence of the infection under sufficiently large disease constant.

Theorem 5.8. The infection is asymptotically permanent for a positive initialization of the infected population on its initialization interval if the disease transmission constant is large enough.

Proof. Note from (5.26) that for any given small $\varepsilon \in \mathbf{R}_{0+}$, there is a sufficiently large finite $T=T(t, \varepsilon) \in \mathbf{R}_{0+}$ such that for any $\sigma \geq T$ and any $t \in \mathbf{R}_{0+}$, one gets

$$
I(t+\sigma) \geq\left(\frac{\beta e^{-b \tau}(1+\delta)}{(\gamma+b+\alpha)\left[\eta+\max \left(u_{S}^{-1}, u_{V}^{-1}\right)\right]}-\varepsilon\right)\left(\inf _{t-2 \tau \leq \xi \leq t-\tau} I(\xi)\right) \geq \inf _{t-2 \tau \leq \xi \leq t-\tau} I(\xi)
$$

if the disease transmission constant is large enough to satisfy

$$
\beta \geq \frac{(1+\varepsilon)(\gamma+b+\alpha) e^{b \tau}\left[\eta+\max \left(u_{S}^{-1}, u_{V}^{-1}\right)\right]}{1+\delta}
$$

If either $u_{S}$ or $u_{V}$ (but not both) is zero, then its inverse is removed from the above condition.

The above results suggest that the infection removal require periodic culling (or partial removal) of the susceptible population through impulsive vaccination so that both populations can become extinguished according to (5.8).

\section{Simulation Results}

This section contains some simulation examples illustrating the theoretical results introduced in Sections 2 and 5 concerning the existence and location of disease-free and endemic equilibrium points under regular and impulsive vaccination as well as the eventual oscillatory behavior. The objective of these examples is to numerically verify the theoretical expressions obtained there. The parameters of the model are: $b=0.05$ (days) $^{-1}, \gamma=0.005$ (days) $^{-1}, 1 / \gamma_{1}=7$ days, $\beta_{1}=\beta / 3$ (days) $^{-1}, \delta=\beta_{1} / \beta, \tau=4$ days, $\eta=0.5, \alpha=0.005$ (days) $^{-1}$, and $\omega=8$ days. The initial conditions are $S(0)=250, V(0)=150, E(0)=150, I(0)=250$, and $R(0)=200$. Firstly, the disease-free case is considered in Section 6.1 while the endemic case will be treated in Sections 6.2 and 6.3. 


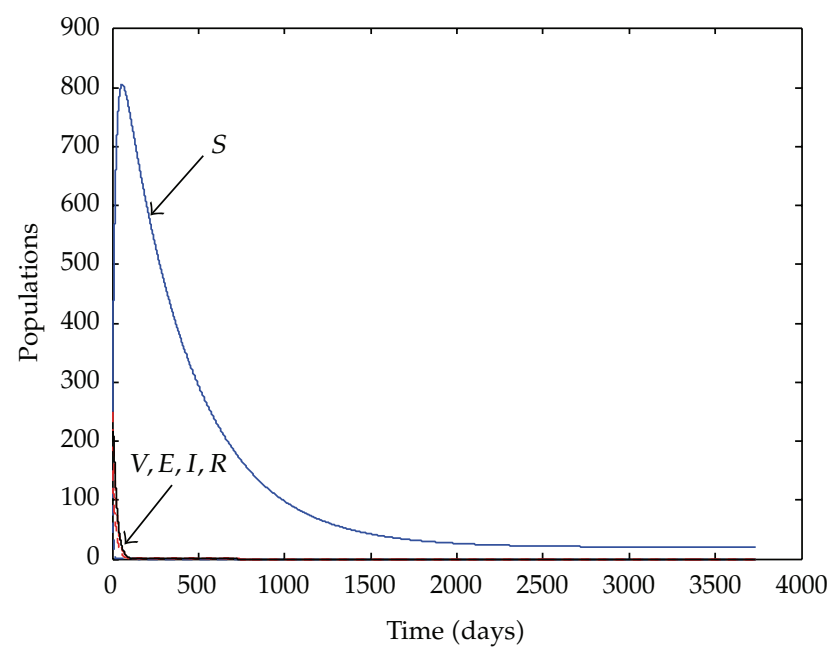

Figure 1: Solution trajectories for $V_{c}=0$.

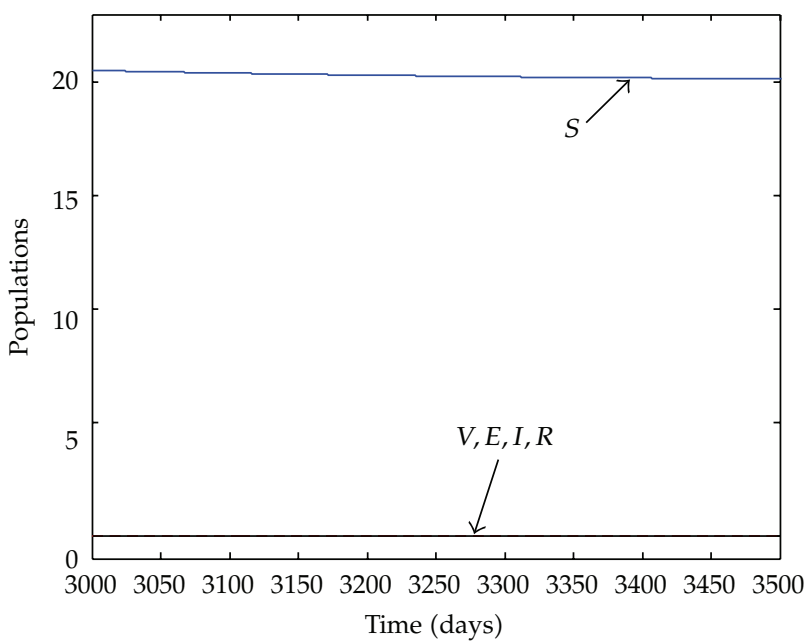

Figure 2: Zoom on the solution trajectories showing the disease-free equilibrium point for $V_{c}=0$.

\subsection{Disease-Free Equilibrium Point}

Consider now $\beta=0.15$ (days) $^{-1}, v=0.95 b$ (days) $^{-1}$ satisfying $v<b$. The two particular cases corresponding to $V_{c}=0$ and $V_{c}=1$ in Section 2 will be studied separately. Thus, the following simulations have been obtained for the SVEIR system (1.1)-(1.5) and $V_{c}=0$.

As it can be appreciated in Figure 1, the vaccinated, exposed, infected, and removedby-immunity (or "immune" or "recovered") population converge to zero and cannot be distinguished in the figure. This situation corresponds to the case when the disease naturally vanishes. On the other hand, the susceptible population presents a different dynamics, reaching a maximum and then converging to a nonzero endemic equilibrium point. Figure 2 shows a zoom on the equilibrium point reached by the model in Figure 1. 


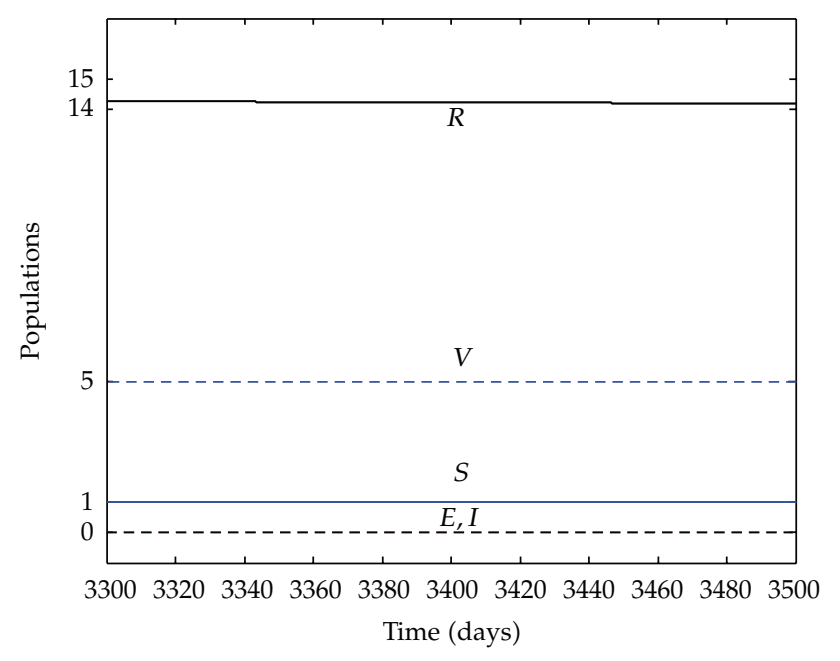

Figure 3: Disease-free equilibrium point when $V_{c}=1$.

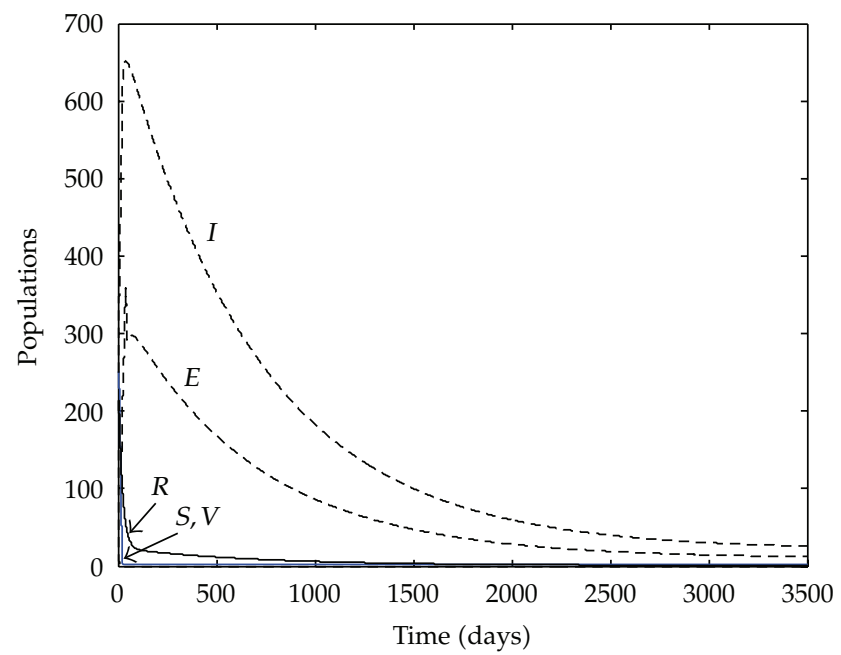

Figure 4: Solution trajectories converging to an endemic equilibrium point.

It can be noted that the vaccinated, exposed, infectious, and removed populations are zero while the susceptible one converges to a number of 20 individuals. Furthermore, those values correspond to the ones stated in Theorem 2.1 for $V_{c}=0$, since all the populations vanish except the susceptible which converges to $S^{*}=b /(b-v)=20$. If $V_{c}=1$, then the solution trajectories converge to the equilibrium point as depicted in Figure 3.

In this case, only the exposed and infected tend to zero while the remaining populations tend to the values calculated in Theorem 2.1 when $V_{c}=1$

$$
S^{*}=1, \quad V^{*}=\frac{v b}{\left(\gamma_{1}+b\right)(b-v)}=4.926, \quad R^{*}=\frac{v \gamma_{1}}{\left(\gamma_{1}+b\right)(b-v)}=14.07, \quad E^{*}=I^{*}=0 .
$$




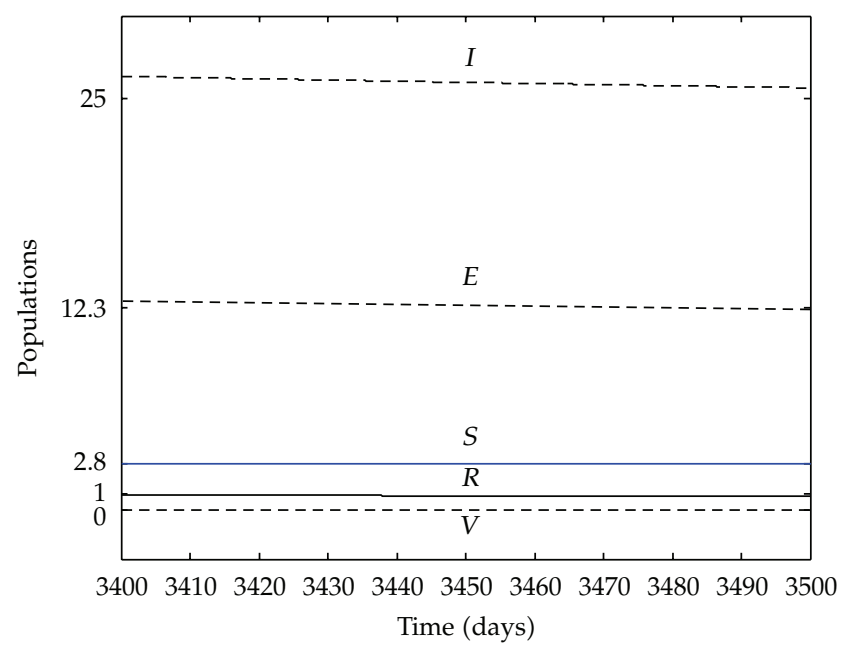

Figure 5: Zoom the solution trajectories showing the endemic equilibrium point.

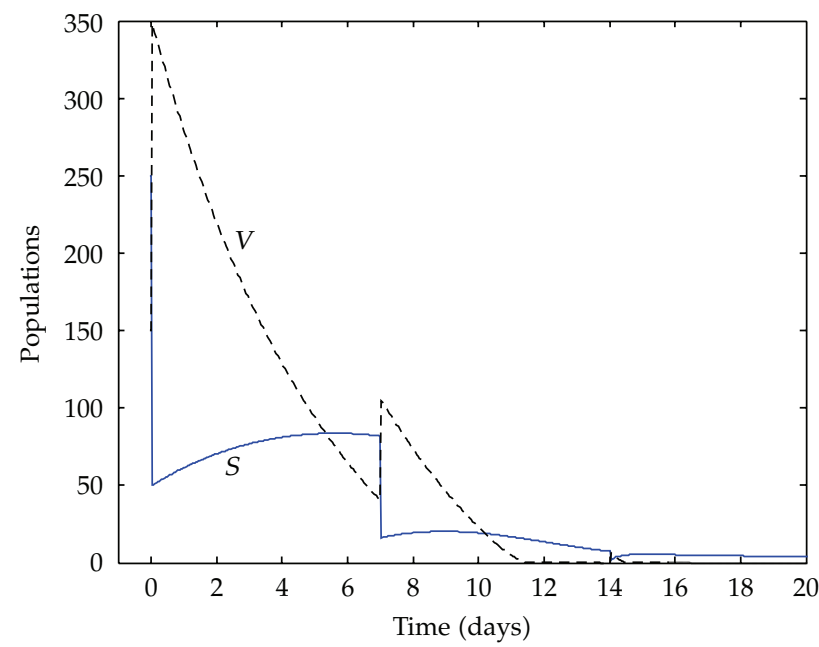

Figure 6: Effect of the impulsive vaccination in susceptible and vaccinated populations.

In the next example, the existence of an endemic equilibrium point is studied through a numerical simulation.

\subsection{Endemic Equilibrium Point}

In order to study the endemic equilibrium point, the value of $\beta$ is changed now to a new value $\beta=1.5$ (days) $^{-1}$ and $v=0.9 b$ (days) $^{-1}$ satisfying the condition $\beta \geq \eta e^{b \tau}(\gamma+b+\alpha)$ stated in Theorem 3.1(i) for $V_{c}=0$. Thus, the system's trajectories are showed in Figure 4 .

A zoom on Figure 4 will show the equilibrium point of the system as represented in Figure 5 .

Figure 5 reveals that there is an endemic equilibrium point, associated to nonzero populations of the exposed and infectious, whose coordinates in view of (5.5) in Section 5 


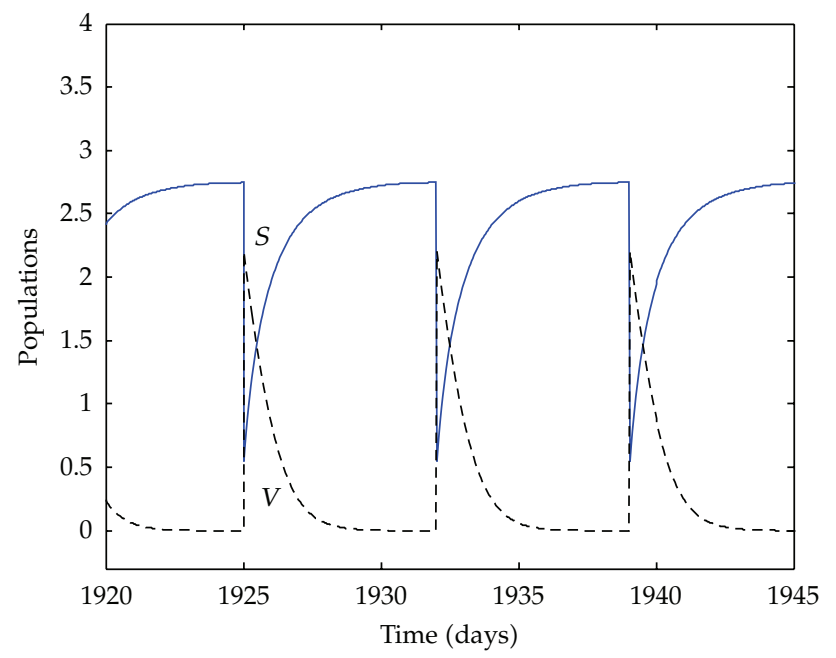

Figure 7: Zoom on the steady-state of susceptible and vaccinated populations.

satisfy the constraints

$$
\begin{gathered}
S^{*}=\frac{\gamma e^{-b \omega} I^{*}+v\left(1-V_{c}\right) N^{*}+b}{b+\beta\left(I^{*} /\left(1+\eta S^{*}\right)\right)}=2.77 ; \quad V^{*}=\frac{v V_{c} N^{*}}{\gamma_{1}+b+\delta \beta\left(I^{*} /\left(1+\eta V^{*}\right)\right)}=0, \\
E^{*}=\frac{\beta}{b}\left(1-e^{-b \omega}\right)\left(\frac{S^{*}}{1+\eta S^{*}}+\frac{\delta V^{*}}{1+\eta V^{*}}\right) I^{*}=12.33, \\
I^{*}=\frac{\beta}{(\gamma+b+\alpha)} e^{-b \tau}\left(\frac{S^{*}}{1+\eta S^{*}}+\frac{\delta V^{*}}{1+\eta V^{*}}\right) I^{*}=25.62, \\
R^{*}=\frac{\gamma_{1} V^{*}+\gamma\left(1-e^{-b \omega}\right) I^{*}}{b}=0.8 .
\end{gathered}
$$

As it can be verified in Figure 5, all the calculated values correspond to the ones obtained in the simulation example. Note that an endemic equilibrium point exists since the exposed and infected are different from zero. The endemic equilibrium point is lost when impulsive vaccination is applied on the system as the next section illustrates.

\subsection{Effect of the Impulsive Vaccination on the Endemic Equilibrium Point}

In this last simulation, the impulsive vaccination considered in Section 5 is introduced in the system. Thus, Equations (5.8) and (5.9) are used, at certain time instants, to remove population from susceptible while increasing the vaccinated. The impulsive vaccination is used once a week (i.e., every 7 days) with $\theta_{k}=\theta=0.8$. The following Figures 6 and 7 show the effect of the impulsive vaccination on the population of susceptible and vaccinated while Figures 8, 9, and 10 show the effect of impulses on the exposed, infected, and immune, 


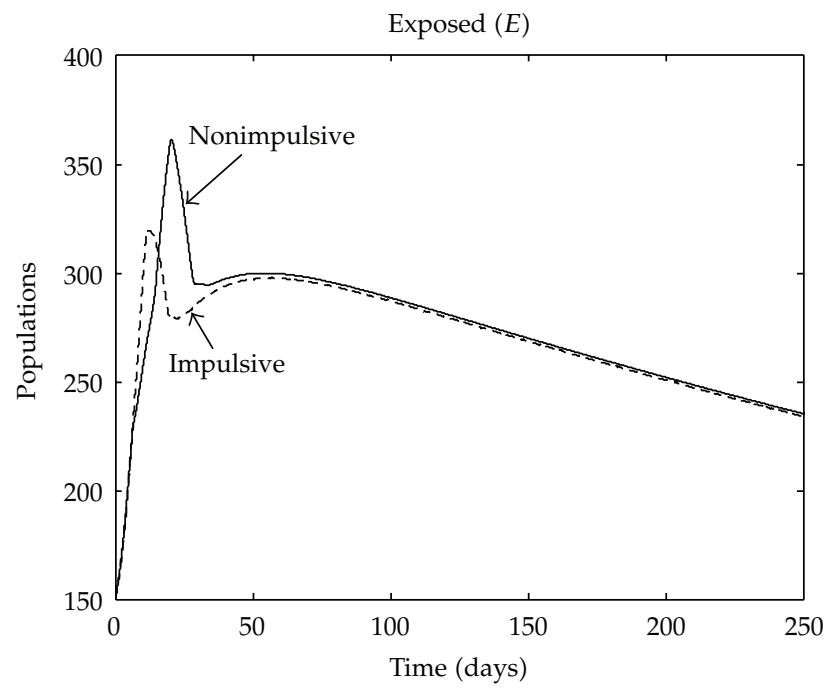

Figure 8: Effect of the impulsive vaccination on exposed.

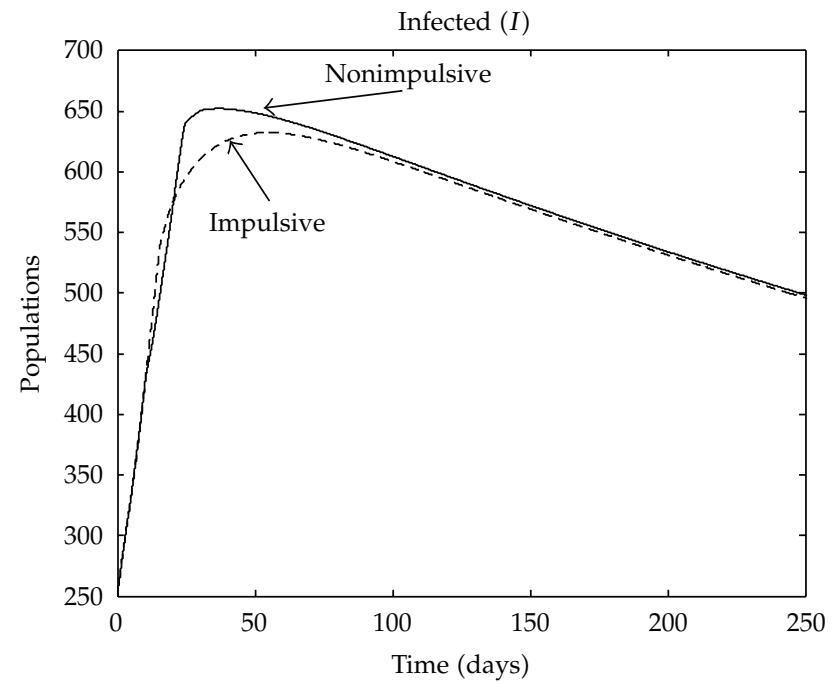

Figure 9: Effect of the impulsive vaccination on infected.

respectively. Notice that the evolution of infected, exposed, and immune is continuous since the impulsive vaccination does not influence directly those populations.

It can be appreciated in Figure 6 that, as expected, the impulsive vaccination reduces the susceptible population in a $20 \%$, that is, such a population is reduced drastically during the first applications of the impulse. On the other hand, Figure 7 depicts the steady-state behavior of the susceptible and vaccinated. In agreement with Theorems 5.4 and 5.5 in Section 5, the endemic equilibrium point is now lost while an oscillatory (periodic indeed) steady-state behavior is obtained. Figures 8,9 , and 10 show the effect of impulse vaccination on the exposed, infected, and immune populations, respectively. It can be observed that the impulse vaccination reduces the maximum peak of the exposed and infected populations 


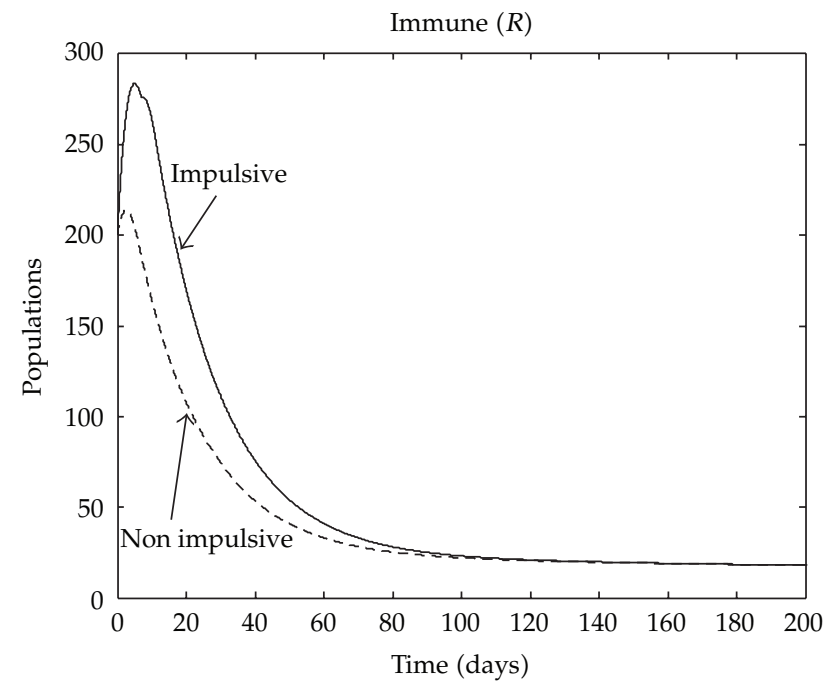

Figure 10: Effect of the impulsive vaccination on the immune population.

while increases the maximum peak of the immune population. However, the solution trajectory solutions are very similar to the nonimpulsive case after the transient. The main reason for this feature relies on the fact that the susceptible population tends to a very small value during the transient and, afterwards, the influence of its variations on the exposed, susceptible, and immune populations is less relevant. Hence, the improvement on the epidemic model state-trajectory solution is concentrated on the transient. In this way, the theoretical results of the manuscript have been illustrated through simulation examples.

\section{Acknowledgments}

The authors thank the Spanish Ministry of Education for the Grant no. DPI2009-07197. They are also grateful to the Basque Government by its support through Grants nos. IT37810, SAIOTEK S-PE08UN15, and SAIOTEK SPE07UN04 and SPE09UN12. The authors are also grateful to the reviewers for their suggestions to improve the former version of the manuscript.

\section{References}

[1] M. De la Sen and S. Alonso-Quesada, "A control theory point of view on Beverton-Holt equation in population dynamics and some of its generalizations," Applied Mathematics and Computation, vol. 199, no. 2, pp. 464-481, 2008.

[2] M. De la Sen and S. Alonso-Quesada, "Control issues for the Beverton-Holt equation in ecology by locally monitoring the environment carrying capacity: non-adaptive and adaptive cases," Applied Mathematics and Computation, vol. 215, no. 7, pp. 2616-2633, 2009.

[3] M. De la Sen and S. Alonso-Quesada, "Model-matching-based control of the Beverton-Holt equation in ecology," Discrete Dynamics in Nature and Society, vol. 2008, Article ID 793512, 21 pages, 2008.

[4] M. De la Sen, "About the properties of a modified generalized Beverton-Holt equation in ecology models," Discrete Dynamics in Nature and Society, vol. 2008, Article ID 592950, 23 pages, 2008.

[5] M. De la Sen, "The generalized Beverton-Holt equation and the control of populations," Applied Mathematical Modelling, vol. 32, no. 11, pp. 2312-2328, 2008. 
[6] D. Mollison, Ed., Epidemic Models: Their Structure and Relation to Data, Publications of the Newton Institute, Cambridge University Press, Cambridge, UK, 1995, transferred to digital printing 2003.

[7] M. J. Keeling and P. Rohani, Modeling Infectious Diseases in Humans and Animals, Princeton University Press, Princeton, NJ, USA, 2008.

[8] A. Yildirim and Y. Cherruault, "Analytical approximate solution of a SIR epidemic model with constant vaccination strategy by homotopy perturbation method," Kybernetes, vol. 38, no. 9, pp. 15661575, 2009.

[9] V. S. Erturk and S. Momani, "Solutions to the problem of prey and predator and the epidemic model via differential transform method," Kybernetes, vol. 37, no. 8, pp. 1180-1188, 2008.

[10] N. Ortega, L. C. Barros, and E. Massad, "Fuzzy gradual rules in epidemiology," Kybernetes, vol. 32, no. 3-4, pp. 460-477, 2003.

[11] H. Khan, R. N. Mohapatra, K. Vajravelu, and S. J. Liao, "The explicit series solution of SIR and SIS epidemic models," Applied Mathematics and Computation, vol. 215, no. 2, pp. 653-669, 2009.

[12] X. Song, Y. Jiang, and H. Wei, "Analysis of a saturation incidence SVEIRS epidemic model with pulse and two time delays," Applied Mathematics and Computation, vol. 214, no. 2, pp. 381-390, 2009.

[13] T. Zhang, J. Liu, and Z. Teng, "Dynamic behavior for a nonautonomous SIRS epidemic model with distributed delays," Applied Mathematics and Computation, vol. 214, no. 2, pp. 624-631, 2009.

[14] B. Mukhopadhyay and R. Bhattacharyya, "Existence of epidemic waves in a disease transmission model with two-habitat population," International Journal of Systems Science. Principles and Applications of Systems and Integration, vol. 38, no. 9, pp. 699-707, 2007.

[15] A. Barreiro and A. Baños, "Delay-dependent stability of reset systems," Automatica, vol. 46, no. 1, pp. 216-221, 2010.

[16] M. De la Sen, "On positivity of singular regular linear time-delay time-invariant systems subject to multiple internal and external incommensurate point delays," Applied Mathematics and Computation, vol. 190, no. 1, pp. 382-401, 2007.

[17] M. de la Sen, "Quadratic stability and stabilization of switched dynamic systems with uncommensurate internal point delays," Applied Mathematics and Computation, vol. 185, no. 1, pp. 508-526, 2007.

[18] Y. Jiang, H. Wei, X. Song, L. Mei, G. Su, and S. Qiu, "Global attractivity and permanence of a delayed SVEIR epidemic model with pulse vaccination and saturation incidence," Applied Mathematics and Computation, vol. 213, no. 2, pp. 312-321, 2009.

[19] X. Song, Y. Jiang, and H. Wei, "Analysis of a saturation incidence SVEIRS epidemic model with pulse and two time delays," Applied Mathematics and Computation, vol. 214, no. 2, pp. 381-390, 2009.

[20] M. De la Sen, R. P. Agarwal, A. Ibeas, and S. Alonso-Quesada, "On a generalized time-varying SEIR epidemic model with mixed point and distributed time-varying delays and combined regular and impulsive vaccination controls," Advances in Difference Equations, vol. 2010, Article ID 281612, 2010.

[21] H. Wei, Y. Jiang, X. Song, G. H. Su, and S. Z. Qiu, "Global attractivity and permanence of a SVEIR epidemic model with pulse vaccination and time delay," Journal of Computational and Applied Mathematics, vol. 229, no. 1, pp. 302-312, 2009.

[22] A. Kaddar, "Stability analysis in a delayed SIR epidemic model with a saturated incidence rate," Nonlinear Analysis: Modelling and Control, vol. 15, no. 3, pp. 299-306, 2010. 